\title{
A Generalization of the Prime Geodesic Theorem to Counting Conjugacy Classes of Free Subgroups
}

\author{
Lewis Bowen *
}

July 9, 2018

\begin{abstract}
The classical prime geodesic theorem (PGT) gives an asymptotic formula (as $x$ tends to infinity) for the number of closed geodesics with length at most $x$ on a hyperbolic manifold $M$. Closed geodesics correspond to conjugacy classes of $\pi_{1}(M)=\Gamma$ where $\Gamma$ is a lattice in $G=S O(n, 1)$. The theorem can be rephrased in the following format. Let $X(\mathbb{Z}, \Gamma)$ be the space of representations of $\mathbb{Z}$ into $\Gamma$ modulo conjugation by $\Gamma . X(\mathbb{Z}, G)$ is defined similarly. Let $\pi: X(\mathbb{Z}, \Gamma) \rightarrow X(\mathbb{Z}, G)$ be the projection map. The PGT provides a volume form vol on $X(\mathbb{Z}, G)$ such that for sequences of subsets $\left\{B_{t}\right\}, B_{t} \subset X(\mathbb{Z}, G)$ satisfying certain explicit hypotheses, $\left|\pi^{-1}\left(B_{t}\right)\right|$ is asymptotic to $\operatorname{vol}\left(B_{t}\right)$.

We prove a statement having a similar format in which $\mathbb{Z}$ is replaced by a free group of finite rank under the additional hypothesis that $n=2$ or 3 .
\end{abstract}

MSC: 20E09, 20F69, 37E35, 51M10

Keywords: subgroup growth, prime geodesic theorem, free subgroup, character variety, hyperbolic group, hyperbolic geometry.

\section{Introduction}

Given a closed manifold $M^{n}$ of contant curvature -1, the prime geodesic theorem states that the number of closed oriented geodesics on $S$ of length at most $L$ is asymptotic to $\frac{e^{(n-1) L}}{(n-1) L}$. This was first proven by [Huber, 1961] (in the $n=2$ case and using Selberg's trace formula) and subsequently generalized by Margulis in his thesis, [Parry and Pollicott, 1983], [Knieper, 1997], [Gunesch, 2005] and others. [Hurt, 2001] and Sharp (see [Margulis, 2004]) have written two recent surveys.

Since $M$ has negative curvature, the set of closed geodesics is naturally in 1-1 correspondence with the set of conjugacy classes of nontrivial elements of $\pi_{1}(M)$. The length of a closed geodesic corresponds to the translation length of any element in its associated conjugacy class. So, the theorem is equivalent to an asymptotic formula for the number of conjugacy classes of the fundamental

\footnotetext{
${ }^{*}$ Research supported in part by a Max Zorn Postdoctoral Fellowship
} 
group counted according to translation length. We will generalize this by providing an asymptotic formula for the number of conjugacy classes of free subgroups counted according to moduli.

\subsection{Example 1: Pairs of Pants}

Before giving a general framework to describe the results, we present an example in the simplest nontrivial case. We assume that $M$ is a closed hyperbolic surface and we are interested in counting conjugacy classes of subgroups $F<\pi_{1}(M)$ where $F$ is free of rank 2. As a subgroup of $\pi_{1}(M), F$ corresponds to a covering space $M_{F} \rightarrow M$. If $F^{\prime}$ is conjugate to $F$ then $M_{F^{\prime}}$ is isometric to $M_{F}$. Since $F$ has rank 2, $M_{F}$ is either an open 3-holed sphere or an open 1-holed torus.

Suppose further that we are only interested in counting conjugacy classes $F$ such that the covering space $M_{F}$ is a 3 -holed sphere. Let $\operatorname{Mod}_{0,3}$ denote the (moduli) space of all isometry classes of complete hyperbolic manifolds which are topologically open 3-holed spheres. There is a natural way to parametrize $\operatorname{Mod}_{0,3}$; there are exactly 3 pairwise nonhomotopic simple closed curves on any 3 -holed sphere. The set of lengths of their geodesic representatives is a parametrization of $\operatorname{Mod}_{0,3}$. So $\operatorname{Mod}_{0,3}=(0, \infty)^{3} / \operatorname{Sym}(3)$ where $\operatorname{Sym}(3)$, the symmetric group on 3 elements acts by permuting the coordinates.

Let $r_{1}, r_{2}, r_{3}$ be distinct positive numbers. One consequence of the main theorem in this paper is that if $\epsilon>0$ then the number of conjugacy classes $[F]$ such that the isometry class of $M_{F}$ has coordinates $\left\{l_{1}, l_{2}, l_{3}\right\} \in \operatorname{Mod}_{0,3}$ satisfying $\left|l_{i}-r_{i} t\right|<\epsilon$ is asymptotic to

$$
\frac{64 \sinh (\epsilon / 2)^{3} e^{\left(r_{1}+r_{2}+r_{3}\right) t / 2}}{\operatorname{vol}\left(T_{1} M\right)}
$$

as $t \rightarrow \infty$. Here $\operatorname{vol}\left(T_{1}(M)\right)=2 \pi(\operatorname{area}(M))=4 \pi^{2} \operatorname{genus}(M)$.

\subsection{The Framework}

Let $G=S O(n, 1)$ which we identify as the group of orientation preserving isometries of the unique complete simply connected space of constant curvature -1 which we will denote by $\mathbb{H}^{n}$. Then $M$ is isometric to $\mathbb{H}^{n} / \Gamma$ for some uniform lattice $\Gamma<G$ and $\Gamma$ is isomorphic to $\pi_{1}(M)$.

Let $F$ be a free group. The representation variety, denoted by $R(F, G)$, is the set of homomorphisms $\phi: F \rightarrow G$. $G$ acts on $R(F, G)$ by conjugation $(g \phi)(f)=g \phi(f) g^{-1}$. The quotient $R(F, G) / G$ is called the character variety and denoted by $X(F, G) . R(F, \Gamma)$ and $X(F, \Gamma)=R(F, \Gamma) / \Gamma$ are defined similarly.

When $F=\mathbb{Z}$ the group of integers, $X(F, \Gamma)=X(\mathbb{Z}, \Gamma)$ is the set of conjugacy classes of cyclic subgroups of $\Gamma$ which is naturally in 1-1 correspondence with the closed geodesics of $M$.

$X(\mathbb{Z}, G)$ is the set of conjugacy classes of cyclic subgroups of $G$. It is well known that $X(\mathbb{Z}, G)$ splits into 3 pairwise disjoint subsets depending on the classification of isometries into elliptic, parabolic and hyperbolic. Hyperbolic isometries are characterized by the property of having exactly 2 fixed points on the boundary at infinity of $\mathbb{H}^{n}$. If $M$ is a closed manifold then all infinite order elements of $\Gamma$ are hyperbolic.

So let $X_{h}(\mathbb{Z}, G) \subset X(\mathbb{Z}, G)$ be the set of conjugacy classes of hyperbolic elements. We identify $X_{h}(\mathbb{Z}, G)$ with $(0, \infty) \times S O(n-2)$ where the first factor accounts for the translation length of the hyperbolic element and the second factor accounts for the holonomy about its axis. Let ool $_{X}$ be the 
measure on $X_{h}(\mathbb{Z}, G) \cong(0, \infty) \times S O(n-2)$ equal to the product of Lebesque measure on $(0, \infty)$ with normalized Haar measure on $S O(n-2)$. The inclusion $\Gamma \rightarrow G$ induces a map $\pi: X(\mathbb{Z}, \Gamma) \rightarrow X(\mathbb{Z}, G)$.

If $B_{t}=[t-\epsilon, t+\epsilon] \times S O(n-2) \subset X_{h}(\mathbb{Z}, G)$ then the prime geodesic theorem is equivalent to the following:

$$
\left|\pi^{-1}\left(B_{t}\right)\right| \sim \int_{B_{t}} \frac{e^{(n-1) x}}{x} \operatorname{dvol}_{X}(x, h) .
$$

Here and everywhere else in this paper $f \sim g$ means $f(t) / g(t) \rightarrow 1$ as $t \rightarrow \infty$.

The main result of this paper generalizes the formula above. It has the following form. Let $F$ be a free group of finite rank and $G=S O(2,1)$ or $S O(3,1)$. Let $\pi: X(F, \Gamma) \rightarrow X(F, G)$ be the map induced by inclusion $\Gamma<G$. For $t>0$, let $B_{t} \subset X(F, G)$. Then, there is an explicit volume form $\operatorname{vol}_{F, \Gamma}$ on $X(F, G)$ such that if certain hypotheses on $\left\{B_{t}\right\}$ are satisfied then $\left|\pi^{-1}\left(B_{t}\right)\right| \sim \operatorname{vol}_{X}\left(B_{t}\right)$. This volume form depends on $\Gamma$ only through the volume of $M$.

\subsection{The Dimension 2 Case}

To be more precise, we must provide appropriate hypotheses on the sequence $\left\{B_{t}\right\}$ and exhibit a formula for $\operatorname{vol}_{F, \Gamma}$. For simplicity, let us first consider the dimension 2 case. We will be concerned only with the subset $X_{d}(F, G) \subset X(F, G)$ of discrete faithful representations with no cusps. The last condition means that if $\phi: F \rightarrow G$ is a representation with conjugacy class $[\phi] \in X_{d}(F, G)$ then $\mathbb{H}^{2} / \phi(F)$ is a surface without cusps; i.e. $\phi(F)$ has no parabolic elements.

So $\mathbb{H}^{2} / \phi(F)$ is a surface of genus $g$ with $n$ holes for some $g$ and $n \geq 1$. The pair $(g, n)$ is invariant under conjugation by $G$ and the character variety $X_{d}(F, G)$ splits as a disjoint union of $X_{g, n}(F, G)$ where $[\phi] \in X_{g, n}(F, G)$ iff $\mathbb{H}^{2} / \phi(F)$ has genus $g$ and $n$ holes. Each connected component of $X_{g, n}(F, G)$ is identifiable with the Teichmuller space of genus $g n$-holed surfaces.

For example, if $F$ has rank 2 then $X_{d}(F, G)=X_{0,3}(F, G) \cup X_{1,1}(F, G)$. Each component of $X_{0,3}(F, G)$ is identifiable with $(0, \infty)^{3}$ which is the universal covering space of $\operatorname{Mod}_{0,3}=(0, \infty)^{3} / \operatorname{Sym}(3)$. In general, $\operatorname{Mod}_{g, n}$ is the quotient of $X_{g, n}(F, G)$ by the group of automorphisms of $F$ which acts on representations by precomposition.

Recall that a pants decomposition of a surface $S$ is a collection $\mathcal{P}=\left\{P_{i}\right\}$ of nonoverlapping embedded 3-holed spheres in $S$ whose union is all of $S$. A pants decomposition determines FenchelNielsen coordinates on the Teichmuller space of $S$ [Casson and Bleiler, 1988]. Roughly speaking, the coordinates are given by a map $F N: \operatorname{Teich}(g, n) \rightarrow \mathbb{R}^{k}$ where $k=6 g+3 n-6$. By pulling back we obtain a volume form and distance function on Teich $(g, n)$ which can then be pulled back to $X_{g, n}(F, G)$. By [Wolpert, 1982] this volume form coincides with the Weil-Petersson volume form. So it is independent of the pants decomposition. We denote it by $v_{W} l_{W}$. The distance function depends on the pants decomposition. We denote it by $d_{\mathcal{P}}$. In section 1.5 below we explain these coordinates in greater detail and extend them to what we call the 'nondegenerate' character varieties $X_{\mathcal{P}}\left(F, \operatorname{Isom}^{+}\left(\mathbb{H}^{n}\right)\right)$ for $n=2,3$.

By definition the convex core of a surface is the smallest convex subset that is homotopy equivalent to the surface. If $[\phi] \in X_{d}(F, G)$, the convex core of $\mathbb{H}^{2} / \phi(F)$ is a compact surface with geodesic boundary. Let $l_{\partial}([\phi])$ denote the total length of this boundary.

For example, if $\mathbb{H}^{2} / \phi(F)$ is an open 3-holed sphere, then its convex core is a compact 3-holed sphere and $l_{\partial}([\phi])=l_{1}+l_{2}+l_{3}$ where $\left\{l_{1}, l_{2}, l_{3}\right\} \in \operatorname{Mod}_{0,3}$ are the coordinates for the isometry class 
of the surface $\mathbb{H}^{2} / \phi(F)$.

If for each $t>0,\left[\phi_{t}\right] \in X_{d}(F, G)$ and $\mathcal{P}_{t}=\left\{P_{t, i}\right\}_{i=1}^{r-1}$ is a pants decomposition of the convex core of $\mathbb{H}^{2} / \phi_{t}(F)$ (where $r$ is the rank of $F$ ) then we call the path $\left\{\left(\phi_{t}, \mathcal{P}_{t}\right)\right\}_{t>0}$ telescoping if the following holds.

- Let $S_{t, i}:=\cup_{j<i} P_{t, j}$. Then $P_{t, i} \cap S_{t, i}$ has either 0,1 or 2 components.

- For all $i>1, \lim _{t \rightarrow \infty} \operatorname{len}\left(\partial S_{t, i}\right)-\operatorname{len}\left(\partial S_{t, i-1}\right)=+\infty$.

As a corollary to the main result we obtain:

Theorem 1.1. Suppose $\left\{\left(\phi_{t}, \mathcal{P}_{t}\right)\right\}_{t>0}$ is telescoping where $\left[\phi_{t}\right] \in X_{g, n}\left(F, \operatorname{Isom}^{+}\left(\mathbb{H}^{2}\right)\right)$. Then

$$
\left|\pi^{-1}\left(N_{\epsilon}\left[\phi_{t}\right]\right)\right| \sim \operatorname{vol}\left(T_{1} M\right)^{1-\operatorname{rank}(F)} 2^{-g} \int_{N_{\epsilon}\left[\phi_{t}\right]} e^{l_{\partial}(\psi) / 2} d \operatorname{vol}_{W P}(\psi)
$$

Here $\pi: X(F, \Gamma) \rightarrow X(F, G)$ is the projection map and $N_{\epsilon}\left[\phi_{t}\right]$ denotes the $\epsilon$-neighborhood of $\left[\phi_{t}\right] \in$ $X_{d}(F, G)$ with respect to the metric $d_{\mathcal{P}_{t}}$.

Remark: The hypotheses are not as restrictive as they might look. In order to obtain any asymptotics of the above sort it is necessary to assume that the length of the shortest closed geodesic of the surface $\mathbb{H}^{2} / \phi_{t}(F)$ tends to infinity. For example if the shortest geodesic of $\mathbb{H}^{2} / \phi_{t}(F)$ is much shorter than the shortest geodesic of $M$ then $\left|\pi^{-1}\left(N_{\epsilon}\left[\phi_{t}\right]\right)\right|=0$. But we will show in section 7 that if the length of the shortest geodesic of $\mathbb{H}^{2} / \phi_{t}(F)$ tends to infinity then there exists a decomposition $\mathcal{P}_{t}$ for which $\left\{\left(\phi_{t}, \mathcal{P}_{t}\right)\right\}_{t>0}$ is telescoping. It is possible that a similar result holds in all dimensions but we were unable to prove it. Our main result proves the above formula for sequences of subsets more general than $\left\{N_{\epsilon}\left[\phi_{t}\right]\right\}$.

\subsubsection{Example 2: One Holed Tori}

If $M$ is a closed hyperbolic surface then how many immersed 1-holed tori are there with geodesic boundary of length $b \in[L-\epsilon, L+\epsilon]$ ?

To answer this, recall that if $F$ is a free group of rank 2 then $X_{1,1}(F, \Gamma)$ is the set of conjugacy classes of faithful homomorphisms $\phi: F \rightarrow \Gamma$ such that the corresponding covering space $M_{F}$ is a one-holed torus. Every component of $X_{1,1}(F, \Gamma)$ is identifiable with the Teichmuller space of the one-holed torus. The Teichmuller space orbifold-covers the moduli space of the one-holed torus. So fix a component $X_{1,1}^{0}(F, \Gamma) \subset X_{1,1}(F, \Gamma)$ and let $Z \subset X_{1,1}^{0}(F, \Gamma)$ be a fundamental domain for the action of the mapping class group on $X_{1,1}^{0}(F, \Gamma)=$ Teich $_{1,1}$. So, under the orbifold cover Teich $_{1,1} \rightarrow \operatorname{Mod}_{1,1}, Z$ projects onto the moduli space $\operatorname{Mod}_{1,1}$ in a 1-1 way everywhere except on its boundary which we may assume is piecewise smooth.

For each $b>0$, let $Z_{b} \subset Z$ be the set of isometry classes of one-holed tori whose boundary has length $b$. If $Y$ is a subset of the real line let $Z_{Y}=\cup_{y \in Y} Z_{y}$. In the language of the above theorem, the number of immersed 1-holed tori with geodesic boundary of length $b \in[L-\epsilon, L+\epsilon]$ equals $\left|\pi^{-1}\left(Z_{[L-\epsilon, L+\epsilon]}\right)\right|$.

We cannot directly apply the theorem above for two reasons. First, the sets $Z_{[L-\epsilon, L+\epsilon]}$ are not in the form of an $\epsilon$-neighborhood. Second, we do not have a telescoping decompositions. 
The first problem can be overcome by appealing to the more general hypotheses of the main theorem (theorem [1.2). This basically amounts to covering $Z_{[L-\epsilon, L+\epsilon]}$ with $\delta$-neighborhoods for some $\delta>0$. To remove the second problem, for $\delta>0$, let $Z_{b}^{\delta}$ be the set of isometry classes of one-holed tori in $Z_{b}$ such that the length of the shortest closed geodesic is at least the product $\delta b$. By the remark above, if for each $t>0,\left[\phi_{t}\right]$ is any arbitrary element of $Z_{t}^{\delta}$ then there exists a pants decomposition $\mathcal{P}_{t}$ such that the 'path' $\left\{\left(\phi_{t}, \mathcal{P}_{t}\right)\right\}$ is telescoping. So we can use the sets $Z_{[L-\epsilon, L+\epsilon]}^{\delta}$ instead of $Z_{[L-\epsilon, L+\epsilon]}$. The volume of $Z_{b}^{\delta}$ divided by the volume of $Z_{b}$ tends to a constant (depending on $\delta$ ) as $b \rightarrow \infty$. This constant tends to 1 as $\delta \rightarrow 0$. Since we are only interested in asymptotics, a diagonal argument implies we can apply the formula directly to $Z_{b}$. So we obtain

$$
\left|\pi^{-1}\left(Z_{[L-\epsilon, L+\epsilon]}\right)\right| \sim \frac{1}{2 \operatorname{vol}\left(T_{1} M\right)} \int_{L-\epsilon}^{L+\epsilon} \operatorname{vol}\left(\operatorname{Mod}_{1,1}(b)\right) e^{b / 2} d b
$$

where $\operatorname{vol}\left(\operatorname{Mod}_{1,1}(b)\right)$ is the Weil-Petersson volume of $\operatorname{Mod}_{1,1}(b)$. According to [Mirzakhani, 2006] $\operatorname{vol}\left(\operatorname{Mod}_{1,1}(b)\right)=b^{2} / 24+\pi^{2} / 6 \sim b^{2} / 24$. Hence

$$
\left|\pi^{-1}\left(Z_{[L-\epsilon, L+\epsilon]}\right)\right| \sim \frac{\sinh (\epsilon / 2) L^{2} e^{L / 2}}{12 \operatorname{vol}\left(T_{1} M\right)} .
$$

For comparison, recall that the number of closed oriented geodesics with length in $[L-\epsilon, L+\epsilon]$ is asymptotic to $\frac{2 \sinh (\epsilon) e^{L}}{L}$.

\subsubsection{Example 3: Two Holed Tori}

Let's assume that $M$ is a closed hyperbolic surface. There is a special reason why someone might want to count immersed two-holed tori in $M$. To explain, we introduce the 2-holed torus graph $G_{M}=(V, E)$ of $M$. The vertex set $V$ of $G_{M}$ is the set of oriented closed geodesics of $M$. There is a directed edge from $v_{1}$ to $v_{2}$ iff there exists an immersed $\pi_{1}$-injective 2-holed torus $T$ such that if $T$ is given the orientation induced by $M$ then the boundary of $T$ is $v_{1} \cup-v_{2}$ (where $-v_{2}$ means $v_{2}$ with the opposite orientation). A directed cycle in $G_{M}$ corresponds to an immersed closed surface $S$ in $M$, obtained by gluing the 2-holed tori together which individually represent edges in the cycle. The fundamental group of $S$ injects into the fundamental group of $M$.

The definition of a 2-holed torus graph can be generalized to the case when $M$ is not a surface except that the vertices then correspond to unoriented geodesics and the edges to (unoriented) immersed $\pi_{1}$-injective 2-holed tori. Cycles still correspond to immersed surfaces but these need not be $\pi_{1}$-injective.

It can be shown using the methods in this paper that every vertex of $G_{M}$ has infinite valence. If $M$ contains an immersed $\pi_{1}$-injective closed surface, then $G_{M}$ necessarily contains directed cycles. It would be interesting to have a more ergodic-theoretic proof that $G_{M}$ contains directed cycles when $M$ is closed surface. Note that path components of $G_{M}$ correspond to homology classes of $M$.

For $L, \epsilon>0$ let $G_{L, \epsilon}$ be the subgraph of $G$ induced by the set of vertices $v$ whose underlying oriented geodesic has length in the interval $[L-\epsilon, L+\epsilon]$.

therefore the

The classical prime geodesic theorem implies that the number of vertices of $G_{L, \epsilon}$ is asymptotic to $2 \sinh (\epsilon) e^{L} / L$. To obtain asymptotics for the number of edges of $G_{L, \epsilon}$ we proceed as in the previous example. 
So fix a component $X_{1,2}^{0}(F, \Gamma) \subset X_{1,2}(F, \Gamma)$ and let $Z \subset X_{1,2}^{0}(F, \Gamma)$ be a fundamental domain for the action of the mapping class group on $X_{1,2}^{0}(F, \Gamma)=$ Teich $_{1,2}$.

For each $\left(b_{1}, b_{2}\right)>0$, let $Z\left(b_{1}, b_{2}\right) \subset Z$ be the set of isometry classes of two-holed tori with geodesic boundary components of length $b_{1}$ and $b_{2}$. If $Y \subset \mathbb{R}^{2}$ let $Z_{Y}=\cup_{y \in Y} Z(y)$. In the language of the above theorem, the number of immersed 2-holed tori with geodesic boundary lengths $\left(b_{1}, b_{2}\right) \in[L-\epsilon, L+\epsilon]^{2}$ equals $\left|\pi^{-1}\left(Z\left([L-\epsilon, L+\epsilon]^{2}\right)\right)\right|$.

As before, we cannot directly apply the theorem above because the sets we are interested in are not $\epsilon$ neighborhoods and do not necessarily have telescoping pants decompositions. But the same tricks used in the previous theorem apply here as well. Therefore

$$
\left|\pi^{-1}\left(Z_{[L-\epsilon, L+\epsilon]^{2}}\right)\right| \sim \frac{1}{2 \operatorname{vol}\left(T_{1} M\right)^{2}} \int_{L-\epsilon}^{L+\epsilon} \int_{L-\epsilon}^{L+\epsilon} \operatorname{vol}\left(\operatorname{Mod}_{1,2}\left(b_{1}, b_{2}\right)\right) e^{\left(b_{1}+b_{2}\right) / 2} d b_{1} d b_{2}
$$

where $\operatorname{vol}\left(\operatorname{Mod}_{1,2}\left(b_{1}, b_{2}\right)\right)$ is the Weil-Petersson volume of $\operatorname{Mod}_{1,2}\left(b_{1}, b_{2}\right)$. According to [Mirzakhani, 2006] $\operatorname{vol}\left(\operatorname{Mod}_{1,2}\left(b_{1}, b_{2}\right)\right)$ is a polynomial of degree 4 in $\left(b_{1}, b_{2}\right)$. The leading coefficients of this polynomial are given by a recursive formula which seems feasible to compute but we did not do it. So all we can say is that there is some constant $C>0$ such that

$$
\left|\pi^{-1}\left(Z\left([L-\epsilon, L+\epsilon]^{2}\right)\right)\right| \sim \frac{C \sinh ^{2}(\epsilon / 2) L^{4} e^{L}}{\operatorname{vol}\left(T_{1}(M)\right)^{2}} .
$$

Therefore, the average degree of a vertex in the graph $G_{L, \epsilon}$ is

$$
\frac{C L^{5} \sinh ^{2}(\epsilon / 2)}{\sinh (\epsilon) \operatorname{vol}\left(T_{1}(M)\right)^{2}} .
$$

It would be interesting to know the degree sequence of this graph.

\subsection{Proof Sketch of Theorem 1.1}

A discrete faithful homomorphism $\phi: F \rightarrow \Gamma$ determines a subgroup $\phi(F)<\Gamma$. Let $\tilde{\phi}: \tilde{S} \rightarrow M$ be the associated covering space. $\tilde{S}$ is naturally endowed with a hyperbolic metric so that $\tilde{\phi}$ is a local isometry. Let $S$ be the convex core of $\tilde{S}$. The map $\tilde{\phi}: S \rightarrow M$ is a locally isometric immersion.

If $\phi^{\prime}$ is $\Gamma$-conjugate to $\phi$, then the induced immersion $\tilde{\phi}^{\prime}: S^{\prime} \rightarrow M$ is related to $\tilde{\phi}: S \rightarrow M$ by an isometry $\Psi: S \rightarrow S^{\prime}$ in the sense that $\tilde{\phi}^{\prime} \circ \Psi=\tilde{\phi}$. Thus up to this natural equivalence relation, a character $[\phi] \in X(F, \Gamma)$ determines a locally isometric immersion $\tilde{\phi}: S \rightarrow M$. The converse is also true. So the problem of counting characters is equivalent to the problem of counting locally isometric immersions.

By decomposing $S$ into pants we see that it suffices to count locally isometric immersions of a pair of pants $P \rightarrow M$ which satisfy various geometric and boundary constraints. To be more precise, we need to answer questions of the following type.

Given a closed geodesic $\gamma$ in $M$, what is the number of immersed pants $P$ in $M$ with one boundary component of $P$ equal to $\gamma$ and such that the other boundary components have lengths in prespecified intervals? Because we also care about twist parameters, we need to be able to count the number of such pants with additional restrictions on the relative positions of the other boundary components with respect to $\gamma$. 
Another type of question we need to answer is as follows. Given two closed geodesics $\gamma_{1}, \gamma_{2} \in M$, what is the number of immersed pants $P$ in $M$ with two of its boundary components equal to $\gamma_{1}$ and $\gamma_{2}$ and such that the other boundary component has length in a prespecified interval? Twist parameters come into play here as well.

To answer both questions observe that a hyperbolic structure on a pair of pants is determined by the lengths of its 3 boundary components. But the hyperbolic structure is also determined by the lengths of just 2 boundary components if the length of the shortest arc between those two is already known. The shortest arc is perpendicular to both components. So rather than counting pants immersions directly, it suffices to count "pairs of eyeglasses"; a pair of eyeglasses is a triple $\left(\sigma_{1}, \sigma_{2}, \gamma\right)$ such that $\sigma_{1}, \sigma_{2}$ are closed geodesics in $M$ and $\gamma$ is a segment that is perpendicular to both $\sigma_{1}$ and $\sigma_{2}$ at its endpoints. Such a triple uniquely determines a locally isometric immersion of a pair of pants into $M$. (Indeed, the pair of pants is homotopy equivalent to a regular neighborhood of $\left.\sigma_{1} \cup \sigma_{2} \cup \gamma\right)$.

Both questions can be further reduced (by a change of variables), to counting the number of perpendiculars between two segments. That is, we now suppose $\sigma_{1}, \sigma_{2}$ are oriented geodesic segments in $M$ and ask how many perpendiculars are there between $\sigma_{1}$ and $\sigma_{2}$ with length in some prespecified interval. It seems possible that this question has appeared elsewhere but we were unable to find it in the literature.

We describe the pertinent result here in a little more detail since it may be of independent interest. We allow $\sigma_{1}$ and $\sigma_{2}$ to depend on a parameter we denote by $L$. For $i=1,2$ let $T_{1}^{\perp} \sigma_{i}$ be the set of unit tangents vectors perpendicular to $\sigma_{i}$. Any segment $\gamma$ perpendicular to $\sigma_{1}$ and $\sigma_{2}$ determines vectors $v_{i}(\gamma) \in T_{1}^{\perp}\left(\sigma_{i}\right)$ tangent to $\gamma$ at its endpoints.

Let $\chi=\chi_{\sigma_{1}, \sigma_{2}}$ be the measure on $T_{1}^{\perp}\left(\sigma_{1}\right) \times T_{1}^{\perp}\left(\sigma_{2}\right) \times[0, \infty)$ defined by setting $\chi(E)$ equal to the number of perpendicular segments $\gamma$ with $\left(v_{1}(\gamma), v_{2}(\gamma)\right.$, length $\left.(\gamma)\right) \in E$. Let $\chi^{\prime}$ be the measure on $T_{1}^{\perp}\left(\sigma_{1}\right) \times T_{1}^{\perp}\left(\sigma_{2}\right) \times[0, \infty)$ with density

$$
d \chi^{\prime}=\frac{e^{\Re(L)}}{2 \operatorname{vol}\left(T_{1} M\right)} d \operatorname{vol}_{T_{1}^{\perp}\left(\sigma_{1}\right)} \operatorname{dvol}_{T_{1}^{\perp}\left(\sigma_{2}\right)} d L .
$$

$\operatorname{vol}_{T_{1}^{\perp}\left(\sigma_{i}\right)}$ is the obvious measure on $T_{1}^{\perp}\left(\sigma_{i}\right)=\sigma_{i} \times S^{0}$ with total mass 2 length $\left(\sigma_{i}\right)$ and $\operatorname{vol}\left(T_{1} M\right)=$ $(2 \pi) \operatorname{area}(M)$. Let $\sigma_{i}^{+}=\sigma_{i} \times\{+1\} \subset T_{1}^{\perp}\left(\sigma_{i}\right)$. We will prove that if $\epsilon>0$ is fixed and length $\left(\sigma_{i}(L)\right) e^{L / 2}$ tends to infinity with $L$ for both $i=1,2$ then

$$
\chi\left(\sigma_{1}^{+} \times \sigma_{2}^{+} \times[L-\epsilon, L+\epsilon]\right) \sim \chi^{\prime}\left(\sigma_{1}^{+} \times \sigma_{2}^{+} \times[L-\epsilon, L+\epsilon]\right) .
$$

Here we use the notation $f(L) \sim g(L)$ to mean $\lim _{L \rightarrow \infty} \frac{f(L)}{g(L)}=1$. An analogous statement holds in dimension 3. See theorem 3.1 for a precise statement.

In summary, we first prove an asymptotic formula for the number of perpendiculars between two segments. Next we introduce detailed notation for describing the geometry of characters in $X\left(\pi_{1}(P), \Gamma\right)$. From this it is easy to answer questions about the number of pants immersions with two boundary components fixed. A longer change of variables argument is necessary to handle the case in which only one boundary component is fixed. The main theorem follows from an inductive argument on the pants decomposition. 


\subsection{Notation and Results for Dimensions 2 and 3}

We will use the following notation throughout the paper. Identify $M$ with the quotient $\mathbb{H}^{n} / \Gamma$ where $\Gamma \equiv \pi_{1}(M)$ is a discrete subgroup of $G=\operatorname{Isom}\left(\mathbb{H}^{n}\right)$. If $n=2$ set $G:=P S L_{2}(\mathbb{R})$. If $n=3$ set $G:=P S L_{2}(\mathbb{C})$. Let $\mathbb{A}:=\mathbb{C} /<2 \pi i>$ and let $\mathbb{F}=\mathbb{R}$ or $\mathbb{A}$ depending on whether $n=2$ or 3 .

\subsubsection{Lengths and Widths}

The length $\operatorname{len}(g) \in \mathbb{F}$ of a hyperbolic element $g \in G$ is defined by

$$
\begin{aligned}
\cosh (\operatorname{len}(g) / 2) & = \pm \text { trace }(g) / 2 \\
\Re(\operatorname{len}(g)) & \geq 0 .
\end{aligned}
$$

Alternatively, $\Re(\operatorname{len}(g))$ is the minimum of $d(x, g x)$ over all $x \in \mathbb{H}^{n}$ where $d(\cdot, \cdot)$ is the distance function. $\Im(\operatorname{len}(g))$ measures the amount of turning along the axis of $g$.

A double cross is a triplet $\left(\sigma_{1}, \sigma_{2} ; \eta\right)$ where $\sigma_{1}, \sigma_{2}, \eta$ are oriented geodesics in $\mathbb{H}^{3}$ such that $\eta$ intersects $\sigma_{1}$ and $\sigma_{2}$ orthogonally Fenchel, W. (1989)]. Let $g \in G$ be such that $g(\eta)=\eta$ and $g\left(\sigma_{1}\right)=\sigma_{2}$. Then the width of $\left(\sigma_{1}, \sigma_{2} ; \eta\right)$ is defined to be $\pm \operatorname{len}(g)$ with the sign determined by:

- if $\sigma_{1} \cap \sigma_{2}=\emptyset$ then the sign is positive iff $\eta$ is directed from $\sigma_{1}$ to $\sigma_{2}$,

- otherwise, the sign is positive iff $\left(\sigma_{1}, \sigma_{2}, \eta\right)$ is a positively oriented frame as determined by the right-hand rule.

\subsubsection{Fenchel-Nielsen Coordinates and a Volume Form on the Character Variety}

Let $S$ be a compact surface with boundary. We will have use for coordinates on $X\left(\pi_{1}(S), G\right)$ that are similar to the Fenchel-Nielsen coordinates [Casson and Bleiler, 1988] on Teichmuller space. To describe these we introduce the notion of an oriented framed pants decomposition.

A pants decomposition of $S$ is a collection $\mathcal{P}=\left\{P_{i}\right\}_{i=1}^{r-1}$ of pairwise nonoverlapping embedded pants $P_{i}$ in $S$ whose union is all of $S . \quad r$ is the rank of the free group $\pi_{1}(S)$. We say that a representation $\phi: \pi_{1}(S) \rightarrow I_{s o m}+\left(\mathbb{H}^{n}\right)$ is nondegenerate with respect to $\mathcal{P}$ iff

- for every $\gamma \in \operatorname{Curve}(\mathcal{P}), \phi([\gamma])$ is a hyperbolic isometry and

- if $\gamma_{1}, \gamma_{2}$ are boundary components of some pair of pants $P \in \mathcal{P}$ and $\left[\gamma_{1}\right],\left[\gamma_{2}\right] \in \pi_{1}(S)$ represent them and freely generate $\pi_{1}(P)<\pi_{1}(S)$ then the axes of $\phi\left(\left[\gamma_{1}\right]\right)$ and $\phi\left(\left[\gamma_{2}\right]\right)$ do not intersect at infinity.

This condition is invariant under conjugation by $G$, so it makes sense to say that a character $[\phi] \in X\left(\pi_{1}(S), G\right)$ is nondegenerate wrt $\mathcal{P}$ iff $\phi$ is nondegenerate wrt $\mathcal{P}$. Let $X_{\mathcal{P}}\left(\pi_{1}(S), G\right)$ denote $\mathcal{P}$-nondegerate characters. Note that $[\phi]$ is nondegenerate wrt $\mathcal{P}$ iff $\phi$ restricted to $P$ is nondegenerate wrt $\{P\}$ for all pants $P \in \mathcal{P}$.

If $\phi$ is faithful and $\phi\left(\pi_{1}(S)\right)$ is discrete and contains no parabolic elements then $\phi$ is nondegenerate with respect to any pants decomposition. To see this, note that the first condition above is automatically satisfied. The second one follows from the fact that if $g_{1}, g_{2} \in \operatorname{Isom}\left(\mathbb{H}^{n}\right)$ are two hyperbolic isometries that share a fixed point then the group generated by $g_{1}$ and $g_{2}$ is either cyclic, nondiscrete, or it contains an elliptic or parabolic isometry. 
For any $i$, any boundary component of $P_{i}$ is called a curve of $\mathcal{P}$. It is a boundary curve if it is also a boundary component of $S$. Otherwise it is an interior curve. Let $\operatorname{Int}(\mathcal{P})$ denote the set of interior curves and $\operatorname{Curve}(\mathcal{P})$ the set of all curves. An orientation on $\mathcal{P}$ is a choice of orientation for each curve of $\mathcal{P}$. Fixing an orientation, a framing of $\mathcal{P}$ is a function $f: \operatorname{Int}(\mathcal{P}) \rightarrow \operatorname{Curve}(\mathcal{P}) \times \operatorname{Curve}(\mathcal{P})$ such that if $P_{1}$ is the pair of pants to left of $\gamma, P_{2}$ is the pair of pants to the right of $\gamma$ and $f(\gamma)=\left(\gamma_{1}, \gamma_{2}\right)$ then $\gamma_{i} \in P_{i}$ for each $i$.

Fix an oriented framed pants decomposition $(\mathcal{P}, f)$. We will define an $\mathbb{A}$-valued twist parameter for each interior curve $\gamma$ with respect to a nondegenerate representation $\phi: \pi_{1}(S) \rightarrow G$ generalizing the usual twist parameter.

Implicit in the definition of $\pi_{1}(S)$ is a basepoint *. After conjugating if necessary, we may assume that $*$ is on the curve $\gamma$. For $i=1,2$, let $\gamma_{i}^{\prime}$ be an oriented closed path based at $*$ freely homotopic to $\gamma_{i}$ in $P_{i}$. Let $\left[\gamma_{i}^{\prime}\right] \in \pi_{1}(S, *)$ represent $\gamma_{i}^{\prime}$. By choosing the paths $\gamma_{i}^{\prime}$ appropriately we may assume that $\left[\gamma_{i}^{\prime}\right]$ and $[\gamma]$ freely generate $\pi_{1}\left(P_{i}, *\right)$.

If $\phi\left(\left[\gamma_{i}^{\prime}\right]\right)$ and $\phi([\gamma])$ are hyperbolic elements of $\operatorname{Isom}\left(\mathbb{H}^{n}\right)$ then let $\tilde{\gamma}_{i}$ be the axis of $\phi\left(\left[\gamma_{i}^{\prime}\right]\right)$ and $\tilde{\gamma}$ the axis of $\phi([\gamma])$.

If $\tilde{\gamma}$ and $\tilde{\gamma}_{i}$ do not share a point on the boundary $\partial_{\infty} \mathbb{H}^{n}$ let $\tilde{\sigma}_{i}$ be the unique geodesic perpendicular to both $\tilde{\gamma}$ and $\tilde{\gamma}_{i}$. We give $\tilde{\sigma}_{i}$ a canonical orientation by requiring that the width $w_{i}$ of $\left(\tilde{\gamma}_{i}, \tilde{\gamma} ; \tilde{\sigma}_{i}\right)$ has nonnegative real part and in case it is pure imaginary, then it has a representative in $[0, \pi] i \subset \mathbb{A}$.

Finally, define the twist parameter twist $_{\phi}(\gamma)=$ width $\left(\tilde{\sigma}_{1}, \tilde{\sigma}_{2} ; \tilde{\gamma}\right)$. It is undefined in degenerate cases but this will not concern us in the sequel.

For the representation $\phi: \pi_{1}(S) \rightarrow G$ define $\operatorname{len}_{\phi}: \operatorname{Curve}(\mathcal{P}) \rightarrow \mathbb{F}$ by $\operatorname{len}_{\phi}(\gamma)=\operatorname{len}(\phi[\gamma])$ where $[\gamma] \in \pi_{1}(S)$ is any element representing $\gamma$. The two functions len $_{\phi}$ and twist $_{\phi}$ are invariant under the action of $G$ by conjugation. Therefore, they give rise to functions $l e n_{[\phi]}$, twist $_{[\phi]}$ that depend only on the conjugacy class $[\phi] \in X_{\mathcal{P}}\left(\pi_{1}(S), G\right)$. Define

$$
\operatorname{len}_{\phi}(\partial S)=\sum_{\gamma \in \operatorname{Curve}(\mathcal{P})-\operatorname{Int}(\mathcal{P})} \operatorname{len}_{\phi}(\gamma)
$$

By definition, $X_{\mathcal{P}}\left(\pi_{1}(S), G\right)$ is the subset of the character variety on which the length and twist functions are well-defined. $X_{\mathcal{P}}\left(\pi_{1}(S), G\right)$ is identified with a subset of $\mathbb{F}^{C u r v e}(\mathcal{P}) \sqcup \operatorname{Int}(\mathcal{P})$ through $[\phi] \rightarrow \operatorname{len}_{[\phi]} \sqcup t$ twist $[\phi]$. Let $\operatorname{vol}_{\mathcal{P}}(\cdot)$ be the pullback of the volume form on $\mathbb{F}^{C u r v e}(\mathcal{P}) \sqcup \operatorname{Int}(\mathcal{P})$.

In the 2-dim case $X_{d}\left(\pi_{1}(S), G\right) \subset X_{\mathcal{P}}\left(\pi_{1}(S), G\right)$ and the restriction of $\operatorname{vol}_{\mathcal{P}}$ to $X_{d}\left(\pi_{1}(S), G\right)$ is the Weil-Petersson volume. We do not know whether a similar statement holds in dimension 3 or in any higher dimension.

\subsubsection{Telescoping Paths in the Character Variety}

Let $\mathcal{P}=\left\{P_{i}\right\}_{i=1}^{r-1}$ be an ordered pants decomposition of $S$. For $t>0$ let $\left[\phi_{t}\right] \in X_{d}\left(\pi_{1}(S), G\right)$. We say that $\left\{\left(\phi_{t}, \mathcal{P}\right)\right\}_{t>0}$ is telescoping if the following holds.

- Let $S_{i}$ be the surface $\cup_{j<i} P_{j}$. Then $P_{i} \cap S_{i}$ has either 0,1 or 2 components.

- For all $i>1, \lim _{t \rightarrow \infty} \operatorname{len}_{\phi_{t}}\left(\partial S_{i}\right)-\operatorname{len}_{\phi_{t}}\left(\partial S_{i-1}\right)=\infty$. 


\subsubsection{Neighborhoods}

Let $\mathcal{P}=\left\{P_{i}\right\}_{i=1}^{r-1}$ be an ordered pants decomposition of $S$. Let $S_{i}$ be as in the previous subsection.

Given a set $E \subset X_{\mathcal{P}}\left(\pi_{1}(S), G\right)$ and an $\epsilon>0$ there are two different $\epsilon$-neighborhoods of the boundary $\partial E=\bar{E}-\operatorname{int}(E)$ that we will consider. The first is $N_{1}^{\mathcal{P}}(\epsilon, \partial E)$ equal to the set of all $\psi \in X_{\mathcal{P}}\left(\pi_{1}(S), G\right)$ such that there exists $\phi \in \partial E$ satisfying the following.

- $l_{\psi}(\gamma)=l_{\phi}(\gamma)$ for all curves $\gamma$ in the decomposition $\mathcal{P}$.

- For any $i$ if $\gamma$ is a curve in $S_{i+1}-S_{i}$ then

$$
\begin{aligned}
& \mid \Re\left(\text { twist }_{\psi}(\gamma)-\text { twist }_{\phi}(\gamma)\right) \mid \leq \epsilon \exp \left(\Re\left(\operatorname{len}_{\psi} \partial S_{i}-\text { len }_{\psi} \partial S_{i+1}\right) / 4\right) \\
& \mid \Im\left(\text { twist }_{\psi}(\gamma)-\text { twist }_{\phi}(\gamma)\right) \mid \leq \epsilon \exp \left(\Re\left(\operatorname{len}_{\psi} \partial S_{i}-\operatorname{len}_{\psi} \partial S_{i+1}\right) / 4\right)
\end{aligned}
$$

The second neighborhood is $N_{2}^{\mathcal{P}}(\epsilon, \partial E)$ equal to the set of all $\psi \in X_{\mathcal{P}}\left(\pi_{1}(S), G\right)$ such that there exists $\phi \in \partial E$ with

- $\left|l e n_{\psi}(\gamma)=l_{\phi}(\gamma)\right|<\epsilon$ for all curves $\gamma$ in the decomposition $\mathcal{P}$,

- twist $_{\gamma}(\psi)=$ twist $_{\gamma}(\phi)$ for all curves $\gamma$ in the decomposition $\mathcal{P}$.

\subsubsection{Statement of the Main Result}

There is a natural projection map $\pi: X\left(\pi_{1}(S), \Gamma\right) \rightarrow X\left(\pi_{1}(S), G\right)$ induced by inclusion $\Gamma<G$. Let $r=\operatorname{rank}\left(\pi_{1}(S)\right)$ and $g=\operatorname{genus}(S)$. If $n=2$ then let $\nu$ be the measure on $X_{\mathcal{P}}\left(\pi_{1}(S), G\right)$ with derivative

$$
d \nu=\operatorname{vol}\left(T_{1} M\right)^{1-r} 2^{-g} e^{\Re\left[l e n_{\psi}(\partial S)\right] / 2} \operatorname{dvol}_{\mathcal{P}}(\psi) .
$$

If $n=3$, define $\nu$ by

$$
d \nu=\operatorname{vol}\left(T_{1} M\right)^{1-r}(2 \pi)^{-r} e^{\Re\left[l n_{\psi}(\partial S)\right]} d \operatorname{vol}_{\mathcal{P}}(\psi)
$$

The main result is:

Theorem 1.2. Let $\mathcal{P}$ be a pants decomposition of $S$ and $\left\{E_{t}\right\}$ a path of subsets $E_{t} \subset X_{\mathcal{P}}\left(\pi_{1}(S), G\right)$. If for every $\left[\phi_{t}\right] \in E_{t}$, the path $\left\{\left(\phi_{t}, \mathcal{P}\right)\right\}_{t>0}$ is telescoping and if

$$
\lim _{t \rightarrow \infty} \frac{\nu\left(N_{1}\left(1, \partial E_{t}\right)\right)}{\nu\left(E_{t}\right)}=0 \quad \text { and } \quad \lim _{\epsilon \rightarrow 0} \limsup _{L \rightarrow \infty} \frac{\nu\left(N_{2}\left(\epsilon, \partial E_{t}\right)\right)}{\nu\left(E_{t}\right)}=0
$$

then $\left|\pi^{-1}\left(E_{t}\right)\right| \sim \nu\left(E_{t}\right)$ as $t$ tends to infinity.

Acknowledgements I'd like to thank Joel Hass for inspiring conversations which helped this work get started. I'd like to thank Chris Judge and Chris Connell for conversations and encouragement on this subject. 


\section{Notations and Conventions}

Throughout the paper, $M^{n}$ denotes a fixed oriented closed hyperbolic $n$-manifold and $T_{1} M$ its unit tangent space. Let $F M$ be the space of positively oriented orthonormal $n$-frames. Each $n$ frame $\left(v_{1}, . ., v_{n}\right) \in T_{1} M \times \ldots \times T_{1} M$ projects to the same point $x \in M$ under the canonical projection from $T_{1} M$ to $M$. The frames $F M$ form a fiber bundle over $T_{1} M$ with a natural projection $\pi: F M \rightarrow T_{1} M$ which selects the first vector in the frame, that is, $\pi\left(v_{1}, . ., v_{n}\right)=v_{1} . \operatorname{Isom}^{+}\left(\mathbb{H}^{n}\right)$ acts on the frame bundle of $\mathbb{H}^{n}$ on the left by

$$
G\left(v_{1}, . ., v_{n}\right)=\left(D G_{x}\left(v_{1}\right), \ldots, D G_{x}\left(v_{n}\right)\right)
$$

where $x$ is the common basepoint of $v_{1}, \ldots, v_{n}$. Fix a reference frame $f_{0} \in \mathbb{H}^{n}$. Identify the frame $G f$ with $G$ itself. Because of this identification, the right action of $\operatorname{Isom}^{+}\left(\mathbb{H}^{n}\right)$ on $F\left(\mathbb{H}^{n}\right)$ is defined by $\left(H f_{0}\right) G=H G f_{0}$ for any $H, G \in I \operatorname{som}^{+}\left(\mathbb{H}^{n}\right)$.

Since $I \operatorname{som}^{+}\left(\mathbb{H}^{n}\right)=F\left(\mathbb{H}^{n}\right), F M$ is identified with $\Gamma \backslash I \operatorname{som}^{+}\left(\mathbb{H}^{n}\right)$ and $M$ itself with $\Gamma \backslash \mathbb{H}^{n}$ where $\Gamma<\operatorname{Isom}^{+}\left(\mathbb{H}^{n}\right)$ is a uniform lattice.

Let $\mathbb{A}=\mathbb{C} /<2 \pi i>$. Let $\mathbb{F}=\mathbb{R}$ or $\mathbb{A}$ depending on whether $n=2$ or $n=3$. For $L \in \mathbb{F}$ let

$$
G_{L}=\left[\begin{array}{cc}
e^{L / 2} & 0 \\
0 & e^{-L / 2}
\end{array}\right] .
$$

If $n=2, G_{L} \in P S L_{2}(\mathbb{R})=\operatorname{Isom}^{+}\left(\mathbb{H}^{2}\right)$. If $n=3, G_{L} \in P S L_{2}(\mathbb{C})=\operatorname{Isom}^{+}\left(\mathbb{H}^{3}\right)$. If $L>0$ is real and positive, the map on $F M$ defined by $f \rightarrow f G_{L}$ is the time $L$ map of the frame flow. Its projection to the unit tangent bundle $T_{1} M$ is the time $L$ map of the geodesic flow.

For $L, \epsilon \in \mathbb{F}$, define $B(L, \epsilon)$ to be the set of $x \in \mathbb{F}$ such that $|\Re(x)-\Re(L)| \leq|\Re(\epsilon)|$ and, in case $n=3, \min _{n \in \mathbb{Z}}|\Im(x)-\Im(L)+2 \pi n| \leq|\Im(\epsilon)|$ where $|\Im(\epsilon)|$ denotes the number in $[0, \pi]$ equal to the distance from $\Im(\epsilon)$ to $0 \bmod 2 \pi$.

We use the notation $f(t) \sim g(t)$ to mean that $\lim _{t \rightarrow \infty} \frac{\Re(f(t))}{\Re(g(t))}=1$ and $\lim _{t \rightarrow \infty} \frac{\Im(f(t))}{\Im(g(t))}=1$.

\section{Counting Perpendiculars}

Let $\sigma_{1}=\sigma_{1}(t), \sigma_{2}=\sigma_{2}(t)$ be two oriented geodesic segments in $M$. We allow them to depend on the parameter $t$ but we often suppress $t$ from the notation.

For $i=1,2, T_{1}^{\perp}\left(\sigma_{i}\right)$ denote the set of unit vectors perpendicular to $\sigma_{i}$. $T_{1}^{\perp}\left(\sigma_{i}\right)$ is naturally identified with $\sigma_{i} \times S^{n-2}$. It carries the product volume form in which the $\sigma_{i}$ factor has total volume length $\left(\sigma_{i}\right)$ and the $S^{n-2}$ has total volume $\Omega_{n-2}$, the volume of the unit $n-2$ sphere.

Any perpendicular $\gamma$ from $\sigma_{1}$ to $\sigma_{2}$ determines vectors $v_{i}(\gamma) \in T_{1}^{\perp}\left(\sigma_{i}\right)$ in the obvious way such that both $v_{1}(\gamma)$ and $v_{2}(\gamma)$ point toward $\gamma$.

Let $\chi=\chi_{\sigma_{1}, \sigma_{2}}$ denote the measure on $T_{1}^{\perp}\left(\sigma_{1}\right) \times T_{1}^{\perp}\left(\sigma_{2}\right) \times \mathbb{F}$ given by setting $\chi(E)$ equal to the number of perpendiculars $\gamma$ from $\sigma_{1}$ to $\sigma_{2}$ such that $\left(v_{1}(\gamma), v_{2}(\gamma)\right.$, len $\left.(\gamma)\right) \in E$ where len $(\gamma)$ denotes the length of $\gamma$ if $n=2$ and $\operatorname{len}(\gamma)$ denotes the width of the double cross $\left(\sigma_{1}, \sigma_{2} ; \gamma\right)$ otherwise. Here $\gamma$ is oriented from $\sigma_{1}$ to $\sigma_{2}$.

Let $\chi^{\prime}$ be the measure on $T_{1}^{\perp}\left(\sigma_{1}\right) \times T_{1}^{\perp}\left(\sigma_{2}\right) \times \mathbb{F}$ with derivative

$$
d \chi^{\prime}=\frac{e^{(n-1) \Re(L)}}{2^{n-1}(2 \pi)^{n-2} \operatorname{vol}\left(T_{1} M\right)} d \operatorname{vol}_{T_{1}^{\perp}\left(\sigma_{1}\right)} d v l_{T_{1}^{\perp}\left(\sigma_{2}\right)} d L
$$


where the measure on $\mathbb{F}$ is standard Lebesgue measure.

Given a set $E \subset T_{1}^{\perp}\left(\sigma_{1}\right) \times T_{1}^{\perp}\left(\sigma_{2}\right) \times \mathbb{F}$ and $\epsilon>0$ there are two different $\epsilon$-neighborhoods of the boundary of $E$ that we will consider. The first is

$$
\begin{aligned}
N_{1}(\epsilon, \partial E):= & \left\{\left(v_{1}, v_{2}, L\right) \in T_{1}^{\perp}\left(\sigma_{1}\right) \times T_{1}^{\perp}\left(\sigma_{2}\right) \times \mathbb{F} ; \exists\left(v_{1}^{\prime}, v_{2}^{\prime}, L\right) \in \partial E\right. \\
& \text { such that } \left.d\left(v_{1}^{\prime}, v_{1}\right) \leq \epsilon e^{-\Re(L) / 2}, d\left(v_{2}^{\prime}, v_{2}\right) \leq \epsilon e^{-\Re(L / 2)}\right\} .
\end{aligned}
$$

In the above we used $d(\cdot, \cdot)$ to denote the usual distance in both $T_{1}^{\perp}\left(\sigma_{1}\right)$ and $T_{1}^{\perp}\left(\sigma_{2}\right)$. The second is

$$
N_{2}(\epsilon, \partial E):=\left\{\left(v_{1}, v_{2}, L\right) \in T_{1}^{\perp}\left(\sigma_{1}\right) \times T_{1}^{\perp}\left(\sigma_{2}\right) \times \mathbb{F} ; \exists\left(v_{1}, v_{2}, L^{\prime}\right) \in \partial E \text { and } d\left(L, L^{\prime}\right) \leq \epsilon\right\} .
$$

We say that a path $\left\{E_{t}\right\} \subset T_{1}^{\perp}\left(\sigma_{1}\right) \times T_{1}^{\perp}\left(\sigma_{2}\right) \times \mathbb{F}$ tends to infinity if

$$
\inf \left\{\Re(l) \mid \exists\left(v_{1}, v_{2}, l\right) \in E_{t}\right\} \rightarrow \infty .
$$

Theorem 3.1. If $\left\{E_{t}\right\} \subset T_{1}^{\perp}\left(\sigma_{1}(t)\right) \times T_{1}^{\perp}\left(\sigma_{2}(t)\right) \times \mathbb{F}$ is a path of compact sets tending to infinity and

$$
\lim _{t \rightarrow \infty} \frac{\chi^{\prime}\left(N_{1}\left(1, \partial E_{t}\right)\right)}{\chi^{\prime}\left(E_{t}\right)}=0 \quad \text { and } \quad \lim _{\epsilon \rightarrow 0} \limsup _{t \rightarrow \infty} \frac{\chi^{\prime}\left(N_{2}\left(\epsilon, \partial E_{t}\right)\right)}{\chi^{\prime}\left(E_{t}\right)}=0
$$

then $\chi\left(E_{t}\right) \sim \chi^{\prime}\left(E_{t}\right)$.

Lemma 3.2. To prove theorem 3.1 it suffices to prove the theorem for 'rectangular' sequences $\left\{E_{t}\right\}$. These are sequences of the form

$$
E_{t}=\mathcal{F}_{1} \times \mathcal{F}_{2} \times B(L, \epsilon) \subset T_{1}^{\perp}\left(\sigma_{1}\right) \times T_{1}^{\perp}\left(\sigma_{2}\right) \times \mathbb{F}
$$

where $\mathcal{F}_{1}, \mathcal{F}_{2}, L$ are functions of $t, \Re(\epsilon)>0$ if $n=2, \pi \geq \Im(\epsilon)>0$ if $n=3, \mathcal{F}_{i}=\mathcal{F}_{i}^{\prime} \times \sigma_{i}, \mathcal{F}_{i}^{\prime}$ is an arc of $S^{n-2}$ with length $\left(\mathcal{F}_{i}^{\prime}\right) e^{L / 2} \rightarrow \infty$ and length $\left(\sigma_{i}\right) e^{L / 2} \rightarrow \infty$.

Proof. Let $\left\{E_{t}\right\}$ be a sequence satisfying the hypotheses of theorem 3.1. Let $\pi: T_{1}^{\perp}\left(\sigma_{1}\right) \times T_{1}^{\perp}\left(\sigma_{2}\right) \times$ $\mathbb{F} \rightarrow \mathbb{F}$ be the projection map. Let $\epsilon>0$ if $n=2$ and $\Re(\epsilon), \operatorname{Im}(\epsilon)>0$ if $n=3$. By partitioning $E_{t}$ if necessary and reparametrizing we may assume that $\pi\left(E_{t}\right) \subset B(L, \epsilon)$. Because

$$
\lim _{t \rightarrow \infty} \frac{\chi^{\prime}\left(N_{1}\left(1, \partial E_{t}\right)\right)}{\chi^{\prime}\left(E_{t}\right)}=0
$$

there is a slowly increasing function $f(t)$ such that $f(t) \rightarrow \infty$ and

$$
\lim _{t \rightarrow \infty} \frac{\chi^{\prime}\left(N_{1}\left(f(t), \partial E_{t}\right)\right)}{\chi^{\prime}\left(E_{t}\right)}=0 .
$$

Subdivide $T_{1}^{\perp}\left(\sigma_{1}\right) \times T_{1}^{\perp}\left(\sigma_{2}\right)$ into rectangular solids with edge length $f(t) e^{-\Re(L) / 2}$. Subdivide $\mathbb{F}$ into rectangles of edge length $\epsilon^{\prime}>0$. Taking the product, we obtain a subdivision of $T_{1}^{\perp}\left(\sigma_{1}\right) \times T_{1}^{\perp}\left(\sigma_{2}\right) \times \mathbb{F}$. Let $E_{t}^{\prime}$ be the union of all those rectangles in the subdivision which nontrivially intersect $E_{t}$. Because of the hypotheses on $E_{t}$,

$$
\lim _{\epsilon^{\prime} \rightarrow 0} \lim _{t \rightarrow \infty} \frac{\chi^{\prime}\left(E_{t}^{\prime}\right)}{\chi^{\prime}\left(E_{t}\right)}=1
$$

So the conclusion of theorem 3.1 is true for $\left\{E_{t}\right\}$ iff it is true for the sequence $\left\{E_{t}^{\prime}\right\}$. But each $E_{t}^{\prime}$ is partioned into rectangles satisfying the hypotheses of this lemma. 
For simplicity of exposition we will only prove the case

$$
E_{t}=T_{1}^{\perp}\left(\sigma_{1}\right) \times T_{1}^{\perp}\left(\sigma_{2}\right) \times B(L, \epsilon)
$$

the general case being similar. By choosing $\epsilon$ smaller if necessary we may assume that $\Re(\epsilon)$ is less than the minimum injectivity radius of $M$.

By a vector pair $b=(x, y)$ we will mean that $x, y \in T_{1}\left(\mathbb{H}^{n}\right)$ (or in $T_{1}(M)$ ) and that they share the same basepoint. We define an action of $\operatorname{Isom}^{+}\left(\mathbb{H}^{n}\right)$ on the space of vector pairs by setting $b \psi=\left(x^{\prime}, y^{\prime}\right)$ if there exists frames $f, f^{\prime}$ with $f=(x, y, \ldots), f^{\prime}=\left(x^{\prime}, y^{\prime}, \ldots\right)$ and $f \psi=f^{\prime}$. The action of $\operatorname{Isom}^{+}\left(\mathbb{H}^{n}\right)$ on the frame bundle is discussed in section 2. Define $-(x, y)=(-x, y)$.

Here is a proof sketch. We consider approximate perpendiculars obtained in the following way. For $i=1,2$, let $b_{i}=\left(x_{i}, y_{i}\right)$ be a vector pair where $x_{i} \in T_{1}^{\perp}\left(\sigma_{i}\right)$ and $y_{i}$ has the same direction as $\sigma_{i}$. Consider $b_{1} G_{L / 2}$ and $b_{2} G_{L / 2}$. If these two vector pairs are close then there is a path $\gamma^{\prime}$ formed by concatenating the two paths traced out by $\left\{x_{i} G_{s} \mid 0 \leq s \leq \Re(L / 2)\right\}(i=1,2)$ with a short segment from the basepoint of $x_{1} G_{L / 2}$ to that of $x_{2} G_{L / 2}$. Depending on how close $b_{1} G_{L / 2}$ and $b_{2} G_{L / 2}$ are, $\gamma^{\prime}$ will be close to a perpendicular $\gamma$ from $\sigma_{1}$ to $\sigma_{2}$. From this procedure we obtain a function $P$ from the set of pairs $\left(b_{1}, b_{2}\right)$ of the above form to the set of perpendiculars from $\sigma_{1}$ to $\sigma_{2}$. Asymptotically, the length of $\gamma$ minus $L$ depends only on the position of $b_{1} G_{L / 2}$ relative to $b_{2} G_{L / 2}$.

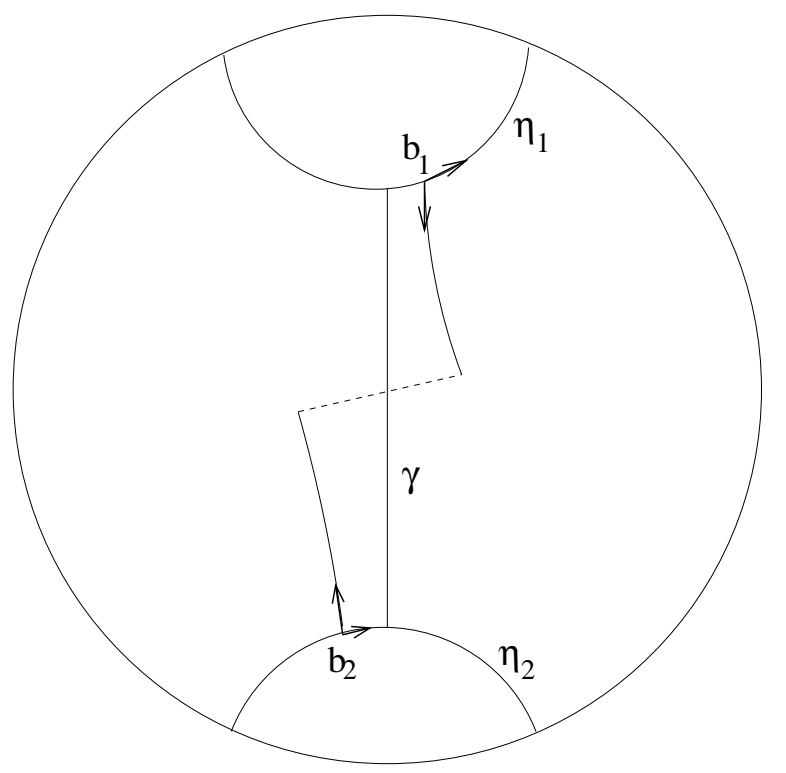

Figure 1: The geodesics $\eta_{1}, \eta_{2}$ and the perpendicular $\gamma$ between them. The 'approximate perpendicular' determined by $b_{1}$ and $b_{2}$ is also shown.

If we choose the pair $\left(b_{1}, b_{2}\right)$ uniformly at random, the distribution of $\left(b_{1} G_{L / 2}, b_{2} G_{L / 2}\right)$ is asymptotically uniform for large $L$. To obtain the asymptotics for $\chi\left(E_{t}\right)$ it suffices to estimate the volume of $P^{-1}(\gamma)$ for each perpendicular $\gamma$. This will be done by relating the calculations to known results regarding the lattice point problem.

Let $\eta_{1}$ and $\eta_{2}$ be two disjoint oriented geodesics in $\mathbb{H}^{n}$. These will play the role of lifts of $\sigma_{1}, \sigma_{2}$ 
to $\mathbb{H}^{n}$ in the sequel. Let $\gamma$ be the perpendicular from $\eta_{1}$ to $\eta_{2}$. For $i=1,2$, let $b_{i}$ be the vector pair $\left(x_{i}, y_{i}\right)$ where $x_{i} \in T_{1}^{\perp}\left(\eta_{i}\right)$ and $y_{i}$ points in the direction of $\eta_{i}$.

Fix $L \in \mathbb{F}$ and let $\phi \in I \operatorname{som}^{+}\left(\mathbb{H}^{n}\right)$ be such that

$$
b_{1} G_{L / 2} \phi=-\left(b_{2} G_{L / 2}\right) .
$$

The pair $(L / 2, \phi)$ determines the pair $\left(b_{1}, b_{2}\right)$ up to the diagonal left action of $\operatorname{Isom}^{+}\left(\mathbb{H}^{n}\right)$. Therefore, $(L / 2, \phi)$ determines the oriented geodesics $\eta_{1}, \eta_{2}$ and $\gamma$ up to rigid motion. By definition, $\eta_{i}$ is the geodesic tangent to $y_{i}$ and $\gamma$ is the perpendicular from $\eta_{1}$ to $\eta_{2}$. It now makes sense to define $W_{L / 2}(\phi)=w i d t h\left(\eta_{1}, \eta_{2} ; \gamma\right)-L$. In other words $W_{L / 2}(\phi)$ is the error in the "length" of the perpendicular corresponding to $\left(b_{1}, b_{2}\right)$ when equation 1 is satisfied.

If $v$ is a unit vector on $\mathbb{H}^{n}$, let $H(v)$ be the unique horoball for which $v$ is an outer unit normal. Let $\Phi \subset I \operatorname{som}^{+}\left(\mathbb{H}^{n}\right)$ be the set of all isometries $\phi$ such that for each unit vector $v$ on $\mathbb{H}^{n}, H(v)$ and $H(-v \phi)$ intersect trivially.

Lemma 3.3. There exists a continuous function $W_{\infty}: \Phi \rightarrow \mathbb{F}$ such that

$$
\lim _{L \rightarrow \infty} W_{L / 2}(\phi)=W_{\infty}(\phi)
$$

Proof. Fix $\phi \in \Phi$. For $i=1,2$, let $b_{i}^{\prime}=\left(x_{i}^{\prime}, y_{i}^{\prime}\right)$ be a vector pair on $\mathbb{H}^{n}$ so that

$$
b_{1}^{\prime} \phi=-b_{2}^{\prime} .
$$

For $L \in \mathbb{F}$, let $b_{i}(L / 2)=b_{i}^{\prime} G_{-L / 2}$. Equation 1 is satisfied. So let $\eta_{1}=\eta_{1}(L / 2), \eta_{2}=\eta_{2}(L / 2), \gamma=$ $\gamma(L / 2)$ be as in the paragraphs preceding this lemma. That is, if $b_{i}=\left(x_{i}, y_{i}\right)$ then $\eta_{i}$ is tangent to $y_{i}$ and $\gamma$ is perpendicular to $\eta_{1}$ and $\eta_{2}$. Let $C_{i}(L / 2)$ be the radius $\Re(L / 2)$ cylinders surrounding $\eta_{i}(L / 2)$. The basepoint of $b_{i}^{\prime}$ is in $C_{i}(L / 2)$. Let $\alpha_{i}(L / 2)$ be a geodesic perpendicular to $\gamma$ which passes through the intersection point $\gamma \cap C_{i}(L / 2)$ such that $\operatorname{width}\left(\eta_{i}, \alpha_{i} ; \gamma\right)=(-1)^{i-1} L / 2$. Then

$$
\begin{aligned}
\operatorname{width}\left(\alpha_{1}, \alpha_{2} ; \gamma\right) & =\operatorname{width}\left(\eta_{1}, \eta_{2} ; \gamma\right)-\operatorname{width}\left(\eta_{1}, \alpha_{1} ; \gamma\right)-\text { width }\left(\alpha_{2}, \eta_{2} ; \gamma\right) \\
& =\operatorname{width}\left(\eta_{1}, \eta_{2} ; \gamma\right)-L=W_{L / 2}(\phi) .
\end{aligned}
$$

We finish the lemma by taking the limit of both sides as $L \rightarrow \infty$. The unit vector parallel to $\alpha_{i}$ at its intersection point with $\gamma$ is parallel to $y_{i}^{\prime}$ with respect to the Euclidean structure on the cylinder $C_{i}$. As $L$ tends to infinity, $C_{i}(L / 2)$ tends to the horosphere $H_{i}$ for which $x_{i}^{\prime}$ is an outer unit normal. Since $\phi \in \Phi, H_{1}$ and $H_{2}$ intersect trivially. Since $\gamma(L / 2)$ is at right angles to $C_{1}(L / 2)$ and $C_{2}(L / 2), \gamma(L / 2)$ converges to the unique geodesic $\gamma(\infty)$ intersecting both $H_{1}$ and $H_{2}$ at right angles. $\alpha_{i}(L / 2)$ converges to the unique geodesic perpendicular to $\gamma(\infty)$ that passes through the intersection point $\gamma(\infty) \cap H_{i}(L / 2)$ and is parallel to $y_{i}^{\prime}$ in $H_{i}$.

Thus $W_{L / 2}(\phi)$ converges to width $\left(\alpha_{1}(\infty), \alpha_{2}(\infty) ; \gamma(\infty)\right)=: W_{\infty}(\phi)$. Since this double cross depends only on $b_{1}^{\prime}$ and $b_{2}^{\prime}$, it is independent of $L$. Continuous dependence on $\phi$ is clear.

Let $\Phi_{L / 2}=\Phi_{L / 2}(\epsilon)$ be the set of isometries $\phi \in \Phi$ such that $W_{L / 2}(\phi) \in B(0, \epsilon)$ and the distance between $v$ and $v \phi$ is at most $\Re(\epsilon)$ for any vector $v \in T_{1}\left(\mathbb{H}^{n}\right)$. Let $\Phi_{\infty}=\Phi_{\infty}(\epsilon)$ be the set of all $\phi \in \Phi$ such that $W_{\infty}(\phi) \in B(0, \epsilon)$ and $d(v, v \phi) \leq \Re(\epsilon)$ for any vector $v$ on $\mathbb{H}^{n}$. By the above lemma, $\Phi_{L / 2}$ converges to $\Phi_{\infty}$ in the Hausdorff topology. 
For a fixed $\phi \in \Phi$ and $L \in \mathbb{F}$, suppose $\left(b_{1}, b_{2}\right)$ satisfies equation 1 Let $\eta_{1}, \eta_{2}, \gamma$ be as above (i.e. $\eta_{i}$ is the geodesic tangent to $y_{i}$ and $\gamma$ is perpendicular to $\eta_{1}$ and $\left.\eta_{2}\right)$. Let $A_{L / 2}(\phi)$ be the set of all $\left(\bar{b}_{1}, \bar{b}_{2}\right)$ where for $i=1,2, \bar{b}_{i}=\left(\bar{x}_{i}, \bar{y}_{i}\right)$ is a vector pair and

- $\bar{x}_{i} \in T_{1}^{\perp}\left(\eta_{i}\right)$,

- $\bar{y}_{i}$ points in the direction of $\eta_{i}$,

- there exists $\psi \in \Phi_{L / 2}$ with

$$
\bar{b}_{1} G_{L / 2} \psi=-\left(\bar{b}_{2} G_{L / 2}\right) .
$$

We can identify $A_{L / 2}(\phi)$ with a subset of $T_{1}^{\perp}\left(\eta_{1}\right) \times T_{1}^{\perp}\left(\sigma_{2}\right)$ by the map $\left(\bar{b}_{1}, \bar{b}_{2}\right) \rightarrow\left(\bar{x}_{1}, \bar{x}_{2}\right)$. This map is 1-1 since $\bar{y}_{i}$ is uniquely determined by $\bar{x}_{i}$. By definition, the volume of $A_{L / 2}(\phi)$ is its volume in $T_{1}^{\perp}\left(\eta_{1}\right) \times T_{1}^{\perp}\left(\eta_{2}\right)$ with respect to product measure.

Lemma 3.4. There exists a continuous function $V$ on $\Phi_{\infty}$ such that

$$
V(\phi)=\lim _{L \rightarrow \infty} \operatorname{vol}\left(A_{L / 2}(\phi)\right) \cosh (\Re(L) / 2)^{2 n-2} .
$$

Also the function $\phi \rightarrow \operatorname{vol}\left(A_{L / 2}(\phi)\right) \cosh (\Re(L) / 2)^{2 n-2}$ converges to $V$ in $L^{1}\left(\Phi_{\infty}\right)$ as $L \rightarrow \infty$.

Proof. Fix $\phi \in \Phi$. Let $b_{i}^{\prime}, b_{i}, \eta_{i}, \gamma, C_{i}, \alpha_{i}, H_{i}$ be as in the previous lemma. If for some $i=1,2$, two unit vectors $v_{0}, v_{1} \in T_{1}^{\perp}\left(\eta_{i}\right)$ share the same basepoint, then the distance between the basepoints of $v_{0} G_{t}$ and $v_{1} G_{t}$ in $C_{i}(t)$ is $\sinh (t)$ times the angle between them. If two vectors $v_{0}, v_{1} \in T_{1}^{\perp}\left(\eta_{i}\right)$ are parallel along $\eta_{i}$ then the distance between the basepoints of $v_{0} G_{t}$ and $v_{1} G_{t}$ in $C_{i}(t)$ is $\cosh (t)$ times the distance between the basepoints of $v_{0}$ and $v_{1}$. Therefore, if $X \subset T_{1}^{\perp}\left(\eta_{i}\right)$ then

$$
\operatorname{vol}\left(X G_{\Re(L / 2)}\right)=\cosh (\Re(L / 2))^{n-1} \operatorname{vol}(X)+O\left(\operatorname{vol}(X) e^{-(n-1) \Re(L / 2)}\right) .
$$

Thus

$$
\operatorname{vol}\left(A_{L / 2}(\phi) G_{\Re(L / 2)}\right)=\cosh (\Re(L / 2))^{2 n-2} \operatorname{vol}\left(A_{L / 2}(\phi)\right)+O\left(\operatorname{vol}^{2}\left(A_{L / 2}(\phi)\right)\right) .
$$

Here $A_{L / 2}(\phi) G_{\Re(L / 2)}$ refers to the diagonal action of $G_{t}$ on $T_{1}^{\perp}\left(\eta_{1}\right) \times T_{1}^{\perp}\left(\eta_{2}\right)$.

As $L$ tends to infinity, the cylinders $C_{i}(L / 2)$ tend to the horospheres $H_{i}$. Let $T_{1}^{\perp}\left(H_{i}\right)$ be the set of outward unit normals to $H_{i}$. Let $A_{\infty}(\phi)$ be the set of pairs $\left(a_{1}, a_{2}\right) \in T_{1}^{\perp}\left(H_{1}\right)$ times $T_{1}^{\perp}\left(H_{2}\right)$ such that there exists $\psi \in \Phi_{\infty}$ with $a_{1} \psi=-a_{2}$.

By definition, a pair $\left(a_{1}, a_{2}\right) \in A_{L / 2}(\phi) G_{\Re(L / 2)}$ if and only if there exists $\psi \in \Phi_{L / 2}$ such that $a_{1} \psi=-a_{2}$. So $A_{L / 2}(\phi) G_{\Re(L / 2)}$ converges in the Hausdorff topology to $A_{\infty}(\phi)$. Because $A_{L / 2}(\phi)$ is defined by a finite set of real analytic inequalities, the volume of $A_{L / 2}(\phi) G_{\Re(L / 2)}$ converges to the volume of $A_{\infty}(\phi)$. Set $V(\phi):=\operatorname{vol}\left(A_{\infty}(\phi)\right)$. It is clear that $V$ is continous. $L^{1}$-convergence follows from pointwise convergence and the boundedness of the two functions.

For $i=1,2$, let $T_{2}^{\perp}\left(\sigma_{i}\right)$ denote the space of vector pairs $b=(x, y)$ such that $x \in T_{1}^{\perp}\left(\sigma_{i}\right)$ and $y$ has the same direction as $\sigma_{i}$. $T_{2}^{\perp}\left(\sigma_{i}\right)$ is naturally identified with $T_{1}^{\perp}\left(\sigma_{i}\right)$ by the map $b \rightarrow x$. 
Let $T_{2}(M)$ denote the space of all vector pairs $(x, y)$ where $x, y$ are unit vectors in $M$ which share the same basepoint and are orthogonal to each other. $T_{2}(M)$ splits locally as a product $M \times S^{n-1} \times S^{n-2}$. It has a natural volume form induced from its Riemannian structure with total mass $\operatorname{vol}(M) \Omega_{n-1} \Omega_{n-2}$. The standard probability measure on $T_{2}(M)$ is this volume form normalized to have total mass 1.

Lemma 3.5. For $i=1,2$ let $\mu_{i}=\mu_{i, t}$ be the probability measure on $T_{2}^{\perp}\left(\sigma_{i}(t)\right)$ obtained from normalizing the volume form on $T_{1}^{\perp}\left(\sigma_{i}(t)\right)$. If $\cosh (\Re(L(t)) / 2)$ length $\left(\sigma_{i}(t)\right) \rightarrow \infty$ and $L(t) \rightarrow \infty$ as $t \rightarrow \infty$ then the pushforward measure $G_{\Re(L / 2) *}\left(\mu_{i}\right)$ converges in the weak* topology to the standard probability measure on $T_{2}(M)$.

Proof. Let $\lambda_{i}$ be a subsequential weak ${ }^{*}$ limit of $\left\{\left(G_{L / 2}\right)_{*}\left(\mu_{i}\right)\right\}_{t}$. Let $\tilde{\sigma}_{i}$ be a lift of $\sigma_{i}$ to the universal cover $\mathbb{H}^{n}$. Let $C$ be the radius $\Re(L / 2)$ cylinder with axis $\tilde{\sigma}_{i}$. Then $T_{2}^{\perp}\left(\tilde{\sigma}_{i}\right) G_{\Re(L / 2)}$ is the set of vector pairs $b=(x, y)$ with $x$ an outward unit normal to $C$ and $y$ parallel to the axis of $C$. The length of this cylinder is $\cosh (L / 2)$ length $\left(\sigma_{i}\right)$ which tends to infinity with $L$ by assumption. So for any radius $R$, the probability that a point chosen uniformly at random on $C$ is within distance $R$ of the boundary of the cylinder tends to zero as $L$ tends to infinity. As noted before, the geometry of the cylinder tends towards that of a horosphere with increasing $L$. It follows that $\lambda_{i}$ is invariant under a rank $n-1$ parabolic subgroup of $\operatorname{Isom}^{+}\left(\mathbb{H}^{n}\right)$. Ratner's theorems Ratner, M. (1991) on unipotent flows now imply the lemma.

Lemma 3.6. Suppose that for $i=1,2$, length $\left(\sigma_{i}(t)\right) \cosh (L(t) / 2) \rightarrow \infty$ as $t \rightarrow \infty$. Then

$$
\chi\left(T_{1}^{\perp}\left(\sigma_{1}\right) \times T_{1}^{\perp}\left(\sigma_{2}\right) \times B(L, \epsilon)\right) \sim c_{n}(\epsilon) e^{(n-1) \Re(L)} \frac{\operatorname{length}\left(\sigma_{1}\right) \operatorname{length}\left(\sigma_{2}\right)}{\operatorname{vol}(F M)}
$$

where

$$
c_{n}(\epsilon)=2^{2-2 n} \Omega_{n-2}^{2} \int_{\Phi_{\infty}} \frac{1}{V(\phi)} d \phi .
$$

The above integral formula is with respect to Haar measure on $\operatorname{Isom}^{+}\left(\mathbb{H}^{n}\right)$.

Proof. Let $\Delta_{L / 2}$ be the set of all pairs $\left(b_{1}, b_{2}\right)$ in which $b_{i}=\left(x_{i}, y_{i}\right) \in T_{2}^{\perp}\left(\sigma_{i}\right)$ and there exists $\phi \in \Phi_{L / 2}$ such that

$$
b_{1} G_{L / 2} \phi=-\left(b_{2} G_{L / 2}\right) .
$$

Associated to every such pair $\left(b_{1}, b_{2}\right)$ is a geodesic segment $\gamma$ perpendicular to $\sigma_{1}$ and $\sigma_{2}$ at its endpoints defined as follows.

The distance from $x_{1} G_{L / 2}$ to $x_{2} G_{L / 2}$ is at most $\Re(\epsilon)$ since $\phi \in \Phi_{L / 2}(\epsilon)$. Let $\gamma^{\prime}$ be the path formed from concatening the segments formed from the trace of $\left\{x_{i} G_{t}: 0 \leq t \leq L / 2\right\}$ (for $i=1,2$ ) with a segment of length at most $\Re(\epsilon)$ joining the basepoint of $x_{1} G_{L / 2}$ to that of $x_{2} G_{L / 2}$. The middle segment is unique since by hypothesis the minimum injectivity radius of $M$ is at least $\Re(\epsilon)$. Let $\tilde{\gamma}^{\prime}$ be a lift of $\gamma^{\prime}$ to the universal cover and let $\tilde{\sigma}_{1}, \tilde{\sigma}_{2}$ be the lifts of $\sigma_{1}, \sigma_{2}$ that pass through the endpoints of $\tilde{\gamma}^{\prime}$. Let $\tilde{\gamma}$ be the common perpendicular to $\tilde{\sigma}_{1}$ and $\tilde{\sigma}_{2}$ and $\gamma$ its projection to $M$.

The definition of $\Phi_{L / 2}(\epsilon)$ implies $\operatorname{len}(\gamma) \in B(L, \epsilon)$. Define $P\left(b_{1}, b_{2}\right)=\gamma$. Conversely, if $\gamma$ is a segment perpendicular to $\sigma_{1}$ and $\sigma_{2}$ at its endpoints and $\operatorname{len}(\gamma) \in B(L, \epsilon)$ then there exists 
$\left(b_{1}, b_{2}\right) \in \Delta_{L / 2}$ such that $P\left(b_{1}, b_{2}\right)=\gamma$. To see this, let $b_{i}=\left(x_{i}, y_{i}\right) \in T_{2}^{\perp}\left(\sigma_{i}\right)$ where $x_{i}=v_{i}(\gamma)$ is as defined in the paragraphs before theorem 3.1. Then $\gamma^{\prime}=\gamma$ and $P\left(b_{1}, b_{2}\right)=\gamma$.

Let $\left(b_{1}, b_{2}\right) \in \Delta_{L / 2}$ be chosen uniformly at random. Let $\phi \in \Phi_{L / 2}$ be such that equation 3 holds. Let $V\left(b_{1}, b_{2}\right)$ denote the volume of

$$
\left\{\left(b_{1}^{\prime}, b_{2}^{\prime}\right) \in \Delta_{L / 2}: P\left(b_{1}, b_{2}\right)=P\left(b_{1}^{\prime}, b_{2}^{\prime}\right)\right\} .
$$

So $V\left(b_{1}, b_{2}\right)=\operatorname{vol}\left(A_{L / 2}(\phi)\right)$ where $A_{L / 2}(\cdot)$ is defined as before lemma 3.4 with $\tilde{\sigma}_{i}$ replacing $\eta_{i}$. Note

$$
\chi\left(T_{1}^{\perp}\left(\sigma_{1}\right) \times T_{1}^{\perp}\left(\sigma_{2}\right) \times B(L, \epsilon)\right)=\operatorname{vol}\left(\Delta_{L / 2}\right) \mathbb{E}\left[1 / V\left(b_{1}, b_{2}\right)\right]
$$

where $\mathbb{E}$ denotes expected value. We claim

$$
\operatorname{vol}\left(\Delta_{L / 2}\right) \sim \Omega_{n-2}^{2} \text { length }\left(\sigma_{1}\right) \text { length }\left(\sigma_{2}\right) \frac{\operatorname{vol}\left(\Phi_{\infty}\right)}{\operatorname{vol}(F M)} .
$$

To see this note that the total mass of $T_{2}^{\perp}\left(\sigma_{1}\right) \times T_{2}^{\perp}\left(\sigma_{2}\right)$ is $\Omega_{n-2}^{2}$ length $\left(\sigma_{1}\right) \operatorname{length}\left(\sigma_{2}\right)$. So

$$
\operatorname{vol}\left(\Delta_{L / 2}\right) / \Omega_{n-2}^{2} \text { length }\left(\sigma_{1}\right) \text { length }\left(\sigma_{2}\right)
$$

is the probability that a pair $\left(b_{1}, b_{2}\right)$ chosen uniformly at random in $T_{2}^{\perp}\left(\sigma_{1}\right) \times T_{2}^{\perp}\left(\sigma_{2}\right)$ is in $\Delta_{L / 2}$. By lemma 3.5 that is asympotic to the probability that a uniformly random pair $\left(b_{1}^{\prime}, b_{2}^{\prime}\right) \in T_{2} M \times T_{2} M$ satisfies $b_{1}^{\prime} \psi=b_{2}^{\prime}$ for some $\psi \in \Phi_{L / 2}(\epsilon)$. The latter equals $\operatorname{vol}\left(\Phi_{L / 2}\right) / \operatorname{vol}(F M)$ which is asymptotic to $\operatorname{vol}\left(\Phi_{\infty}\right) / \operatorname{vol}(F M)$.

By lemma 3.4. $V\left(b_{1}, b_{2}\right)$ is asymptotic to $V(\phi) \cosh ^{2-2 n}(\Re(L) / 2)$ where $\phi \in \operatorname{Isom}^{+}\left(\mathbb{H}^{n}\right)$ is any element such that equation 3 holds. Lemma 3.5 implies

$$
\mathbb{E}\left[1 / V\left(b_{1}, b_{2}\right)\right] \sim \frac{1}{\operatorname{vol}\left(\Phi_{\infty}\right)} \int_{\Phi_{\infty}} \frac{1}{V(\phi) \cosh ^{2-2 n}(\Re(L) / 2)} d \phi .
$$

So,

$$
\begin{aligned}
\chi\left(T_{1}^{\perp}\left(\sigma_{1}\right) \times T_{1}^{\perp}\left(\sigma_{2}\right) \times B(L, \epsilon)\right) & =\operatorname{vol}\left(\Delta_{L / 2}\right) \mathbb{E}\left[1 / V\left(b_{1}, b_{2}\right)\right] \\
& \sim c_{n}(\epsilon) e^{(n-1) \Re(L)} \frac{\text { length }\left(\sigma_{1}\right) \text { length }\left(\sigma_{2}\right)}{\operatorname{vol}(F M)}
\end{aligned}
$$

where

$$
c_{n}(\epsilon)=2^{2-2 n} \Omega_{n-2}^{2} \int_{\Phi_{\infty}} \frac{1}{V(\phi)} d \phi .
$$

The next step in the proof of theorem 3.1 is to compute $c_{n}(\epsilon)$. The previous lemmata can all be modified slightly by replacing the segments $\eta_{i}$ and $\sigma_{i}$ with points. The resulting lemmas apply to the lattice point problem. The value of $c_{n}(\epsilon)$ will then follow from classical results.

For $L, \epsilon_{1}$ positive real numbers and $p_{1}, p_{2} \in M$, let $N\left(p_{1}, p_{2}, L, \epsilon_{1}\right)$ be the set of geodesic segments from $p_{1}$ to $p_{2}$ with length in the interval $\left(L-\epsilon_{1}, L+\epsilon_{1}\right)$. The analogue of lemma 3.6 is: 


\section{Lemma 3.7.}

$$
\left|N\left(p_{1}, p_{2}, L, \epsilon_{1}\right)\right| \sim c_{n}^{\prime}\left(\epsilon_{1}+i \pi\right) e^{(n-1) L} \operatorname{vol}(M)
$$

where

$$
c_{n}^{\prime}(\epsilon)=\frac{\Omega_{n-1}^{2} c_{n}(\epsilon)}{\Omega_{n-2}^{2}} .
$$

Before proving the above, we need analogues for lemmas [3.3] 3.4] and [3.5. Let $v_{1}, v_{2}$ be unit vectors on $\mathbb{H}^{n}$. Suppose $\phi \in I \operatorname{som}^{+}\left(\mathbb{H}^{n}\right)$ satisfies

$$
v_{1} G_{L / 2} \phi=-\left(v_{2} G_{L / 2}\right) .
$$

The pair $\left(v_{1}, v_{2}\right)$ is determined up to a rigid motion by $L, \phi$ and the above equation. So it makes sense to define $W_{L / 2}^{\prime}(\phi)$ to be the distance between the basepoints of $v_{1}$ and $v_{2}$ minus $L$.

Lemma 3.8. If $\phi \in \Phi$ then

$$
\lim _{L \rightarrow \infty} W_{L / 2}^{\prime}(\phi)=\Re\left(W_{\infty}(\phi)\right) .
$$

where $W_{\infty}$ is as defined in lemma 3.3.

Proof. The proof is similar to the proof of lemma 3.3. The cylinders $C_{i}(L / 2)$ are replaced with spheres $S_{i}(L / 2)$ of radius $L / 2$ centered at the basepoint of $v_{i}$.

Let $\Phi_{L / 2}^{\prime}=\Phi_{L / 2}^{\prime}(\epsilon)$ be the set of isometries $\phi \in \Phi$ satisfying:

- $W_{L / 2}^{\prime}(\phi) \in\left(-\epsilon_{1}, \epsilon_{1}\right)$,

- for any vector $v \in T_{1}\left(\mathbb{H}^{n}\right)$, the distance between $v$ and $v \phi$ is at most $\epsilon$.

As before, $\Phi_{\infty}$ is defined as above except that the first condition is replaced by $W_{\infty}(\phi) \in\left(-\epsilon_{1}, \epsilon_{1}\right)$. Lemma 3.8 implies that $\Phi_{L / 2}^{\prime}$ converges to $\Phi_{\infty}$ in the Hausdorff topology.

For a fixed $\phi \in \Phi$ and $L \in \mathbb{F}$, suppose $\left(v_{1}, v_{2}\right)$ satisfies equation 4 . For $i=1,2$, let $q_{i}$ be the basepoint of $v_{i}$. Let $A_{L / 2}^{\prime}(\phi)$ be the set of all $\left(z_{1}, z_{2}\right) \in T_{1}\left(q_{1}\right) \times T_{1}\left(q_{2}\right)$ such that there exists $\psi \in \Phi_{L / 2}^{\prime}$ with

$$
z_{1} G_{L / 2} \psi=-\left(z_{2} G_{L / 2}\right) .
$$

Lemma 3.9. With $V: \Phi_{\infty} \rightarrow \mathbb{R}$ defined as in lemma 3.4.

$$
V(\phi)=\lim _{L \rightarrow \infty} \operatorname{vol}\left(A_{L / 2}^{\prime}(\phi)\right) \cosh (L / 2)^{2 n-2}
$$

for all $\phi \in \Phi_{\infty}$. Convergence also holds in $L^{1}\left(\Phi_{\infty}\right)$.

Proof. The proof is similar to the proof of 3.4 . 
Lemma 3.10. For $i=1,2$ let $\mu_{i}^{\prime}$ be the obvious probability measure on $T_{1}\left(q_{i}\right)$. The pushforward measure $\left\{G_{L / 2 *}\left(\mu_{i}\right)\right\}_{L}$ converges in the weak* topology to the standard probability measure on $T_{1} M$ obtained by normalizing its volume form.

Proof. This result is classical. See [Bekka and Mayer, 2000] for example.

Proof. (of lemma 3.7) The proof is similar to the proof of lemma 3.6 Let $\Delta_{L / 2}^{\prime}$ be the set of all pairs $\left(v_{1}, v_{2}\right) \in T_{1}\left(p_{1}\right) \times T_{1}\left(p_{2}\right)$ such that there exists $\phi \in \Phi_{L / 2}^{\prime}$ such that

$$
v_{1} G_{L / 2} \phi=-\left(v_{2} G_{L / 2}\right) .
$$

For $\left(v_{1}, v_{2}\right) \in \Delta_{L / 2}^{\prime}$ let $P\left(v_{1}, v_{2}\right)$ denote the corresponding segment from $p_{1}$ to $p_{2}$. Let $V\left(v_{1}, v_{2}\right)$ denote the volume of the set of all $\left(v_{1}^{\prime}, v_{2}^{\prime}\right) \in \Delta_{L / 2}^{\prime}$ with $P\left(v_{1}^{\prime}, v_{2}^{\prime}\right)=P\left(v_{1}, v_{2}\right)$. As in lemma 3.6.

$$
\left|N\left(p_{1}, p_{2}, L, \epsilon_{1}\right)\right|=\operatorname{vol}\left(\Delta_{L / 2}^{\prime}\right) \mathbb{E}\left[1 / V\left(v_{1}, v_{2}\right)\right]
$$

where $\mathbb{E}$ denotes expected value. Since $\operatorname{vol}\left(T_{1}\left(p_{1}\right) \times T_{1}\left(p_{2}\right)\right)=\Omega_{n-1}^{2}$ it follows from lemma 3.10 that

$$
\operatorname{vol}\left(\Delta_{L / 2}^{\prime}\right) \sim \frac{\Omega_{n-1}^{2} \operatorname{vol}\left(\Phi_{\infty}\right)}{\operatorname{vol}(F M)}
$$

By lemma 3.9 $V\left(v_{1}, v_{2}\right)$ is asymptotic to $V(\phi) \cosh ^{2-2 n}(\Re(L) / 2)$ where $\phi \in \operatorname{Isom}^{+}\left(\mathbb{H}^{n}\right)$ is any element satisfying equation [6. So

$$
\mathbb{E}\left[1 / V\left(v_{1}, v_{2}\right)\right] \sim \frac{1}{\operatorname{vol}\left(\Phi_{\infty}\right)} \int_{\Phi_{\infty}} \frac{1}{V((\phi)) \cosh ^{2-2 n}(\Re(L) / 2)} d \phi .
$$

So

$$
\begin{aligned}
\left|N\left(p_{1}, p_{2}, L, \epsilon_{1}\right)\right| & =\operatorname{vol}\left(\Delta_{L / 2}^{\prime}\right) \mathbb{E}\left[1 / V\left(b_{1}, b_{2}\right)\right] \\
& \sim c_{n}^{\prime}(\epsilon) e^{(n-1) \Re(L)} \frac{1}{\operatorname{vol}(F M)}
\end{aligned}
$$

where

$$
c_{n}^{\prime}(\epsilon)=2^{2-2 n} \Omega_{n-1}^{2} \int_{\Phi_{\infty}} \frac{1}{V(\phi)} d \phi=\frac{\Omega_{n-1}^{2} c_{n}(\epsilon)}{\Omega_{n-2}^{2}} .
$$

Lemma 3.11. If $n=2$, let $\epsilon>0$. Otherwise let $\epsilon=\epsilon_{1}+i \epsilon_{2} \in \mathbb{A}$ with $\epsilon_{1}>0$ and $0<\epsilon_{2} \leq \pi$. Then

$$
c_{n}(\epsilon)=\left\{\begin{array}{ll}
\frac{\Omega_{n-2}^{2} \sinh (\epsilon) \operatorname{vol}(F M)}{\Omega_{n-1}(n-1) 2^{n-2} \operatorname{vol}(M)} & \text { if } n=2 \\
\frac{2 \epsilon_{2}}{2 \pi} \frac{\Omega_{n-2}^{2} \sinh \left((n-1) \epsilon_{1}\right) \operatorname{vol}(F M)}{\Omega_{n-1}(n-1) 2^{n-2} \operatorname{vol}(M)} & \text { if } n=3 .
\end{array}\right\}
$$


Proof. It is a classical result (Bekka and Mayer, 2000) that

$$
\left|N\left(p_{1}, p_{2}, L, \epsilon_{1}\right)\right| \sim \frac{\operatorname{vol}\left(B_{L+\epsilon_{1}}\right)-\operatorname{vol}\left(B_{L-\epsilon_{1}}\right)}{\operatorname{vol}(M)}
$$

when $L>0$. The volume of $B_{r}^{n}$, the radius $r$ ball in $\mathbb{H}^{n}$, is

$$
\operatorname{vol}\left(B_{r}^{n}\right)=\Omega_{n-1} \int_{0}^{r} \sinh ^{n-1}(t) d t \sim \Omega_{n-1} \frac{\cosh ^{n-1}(r)}{n-1} .
$$

So

$$
\left|N\left(p_{1}, p_{2}, L, \epsilon_{1}\right)\right| \sim \frac{\Omega_{n-1} \sinh \left((n-1) \epsilon_{1}\right) e^{(n-1) L}}{(n-1) 2^{n-2} \operatorname{vol}(M)} .
$$

By the previous lemma,

$$
\left|N\left(p_{1}, p_{2}, L, \epsilon_{1}\right)\right| \sim \frac{c_{n}^{\prime}\left(\epsilon_{1}+i \pi\right) e^{(n-1) \Re(L)}}{\operatorname{vol}(F M)} .
$$

Therefore,

$$
c_{n}^{\prime}\left(\epsilon_{1}+i \pi\right)=\frac{\Omega_{n-1} \sinh \left((n-1) \epsilon_{1}\right) \operatorname{vol}(F M)}{(n-1) 2^{n-2} \operatorname{vol}(M)} .
$$

Now,

$$
c_{n}(\epsilon)=\frac{\Omega_{n-2}^{2}}{\Omega_{n-1}^{2}} c_{n}^{\prime}(\epsilon)=\frac{\Omega_{n-2}^{2} \sinh \left((n-1) \epsilon_{1}\right) \operatorname{vol}(F M)}{\Omega_{n-1}(n-1) 2^{n-2} \operatorname{vol}(M)} .
$$

This proves the cases $n=2$ and $\epsilon_{2}=\pi$. Lemma 3.6 implies that

$$
\chi\left(T_{1}^{\perp}\left(\sigma_{1}\right) \times T_{1}^{\perp}\left(\sigma_{2}\right) \times B(L, \epsilon)\right) \sim \chi\left(T_{1}^{\perp}\left(\sigma_{1}\right) \times T_{1}^{\perp}\left(\sigma_{2}\right) \times B(L+i x, \epsilon)\right)
$$

for any $x \in \mathbb{R}$. So if $\gamma=\gamma_{L}$ is a common perpendicular between $\sigma_{1}$ and $\sigma_{2}$ chosen uniformly at random among all perpendiculars with $|\Re(\operatorname{len}(\gamma))-\Re(L)|<\epsilon$ then the distribution of $\Im(\operatorname{len}(\gamma))$ is asymptotically uniform in $[-\pi, \pi]$. This implies equidistribution of holonomy which finishes the lemma.

Proof. (of theorem 3.1) Assume $\left\{E_{t}\right\}$ is rectangular in the sense of lemma 3.2 In the case when $\mathcal{F}_{1}=T_{1}^{\perp}\left(\sigma_{1}\right), \mathcal{F}_{2}=T_{1}^{\perp}\left(\sigma_{2}\right)$ and $n=3$ it follows from lemma 3.6 and lemma 3.11 above that

$$
\begin{aligned}
\chi\left(E_{t}\right) & \sim \frac{2|\Im(\epsilon)|}{2 \pi} \frac{\Omega_{n-2}^{2} \sinh (\Re((n-1) \epsilon)) \operatorname{vol}(F M)}{\Omega_{n-1}(n-1) 2^{n-2} \operatorname{vol}(M)} e^{(n-1) \Re(L)} \frac{\text { length }\left(\sigma_{1}\right) \text { length }\left(\sigma_{2}\right)}{\operatorname{vol}(F M)} \\
& =\frac{2|\Im(\epsilon)|}{2 \pi} \frac{\sinh (\Re((n-1) \epsilon))}{(n-1) 2^{n-2} \operatorname{vol}\left(T_{1} M\right)} e^{(n-1) \Re(L)} \operatorname{vol}\left(T_{1}^{\perp}\left(\sigma_{1}\right)\right) \operatorname{vol}\left(T_{1}^{\perp}\left(\sigma_{2}\right)\right) .
\end{aligned}
$$

Here we used that $\operatorname{vol}\left(T_{1}^{\perp}\left(\sigma_{i}\right)\right)=\Omega_{n-2} \operatorname{length}\left(\sigma_{i}\right)$ and $\operatorname{vol}\left(T_{1} M\right)=\Omega_{n-1} \operatorname{vol}(M)$. A routine calculation shows that this equals $\chi^{\prime}\left(E_{t}\right)$. The $n=2$ case is similar. The general case is also similar and is left to the reader. 


\section{Pants and Free Subgroups: Definitions and Notation}

We need more notation for pants. Consider the dimension 2 case first. Let $P$ be a pair of pants with a hyperbolic metric and geodesic boundary components $\partial_{1}, \partial_{2}, \partial_{3}$. Let $l_{i}$ be the length of $\partial_{i}$. For $i \neq j$ define $l_{i j}\left(l_{1}, l_{2}, l_{3}\right)$ to be the length of the shortest path from $\partial_{i}$ to $\partial_{j}$. We will give a formula for $l_{12}$ that is asymptotic as all three lengths $l_{1}, l_{2}, l_{3} \rightarrow \infty$.

In the case of dimension 3 , consider a pair of pants $P$ with oriented boundary components $\partial_{i}$ $(i=1,2,3)$. Let $\phi: \pi_{1}(P) \rightarrow \operatorname{Isom}^{+}\left(\mathbb{H}^{3}\right)$ be a homomorphism. Let $*$ be a basepoint in the interior of $P$. Let $\partial_{i}^{\prime}$ be a loop based at* freely homotopic to $\partial_{i}$. By choosing $\partial_{i}^{\prime}$ appropriately we may assume that $\left[\partial_{1}^{\prime}\right]$ and $\left[\partial_{2}^{\prime}\right]$ freely generate $\pi_{1}(P, *)$ and $\left[\partial_{3}^{\prime}\right]=\left[\partial_{1}^{\prime}\right]\left[\partial_{2}^{\prime}\right]$. Define $l_{i}(\phi)=l_{\phi}\left(\partial_{i}\right)=\operatorname{len}\left(\phi\left(\left[\partial_{i}^{\prime}\right]\right)\right)$. If $\phi\left(\left[\partial_{i}^{\prime}\right]\right)$ is a hyperbolic isometry in $\operatorname{Isom}^{+}\left(\mathbb{H}^{3}\right)$ then let $\tilde{\partial}_{i}$ denote its axis.

If for $i \neq j \tilde{\partial}_{i}$ and $\tilde{\partial}_{j}$ do not have a common point at infinity then let $\tilde{\partial}_{i j}$ denote the geodesic perpendicular to $\tilde{\partial}_{i}$ and $\tilde{\partial}_{j}$ oriented from $\tilde{\partial}_{i}$ to $\tilde{\partial}_{j}$. Define $l_{i j}(\phi)=w i d t h\left(\tilde{\partial}_{i}, \tilde{\partial}_{j} ; \tilde{\partial}_{i j}\right)$. If orientations on $\tilde{\partial}_{i}$ and $\tilde{\partial}_{j}$ are not specified, then $l_{i j}(\phi)$ is defined only up to $\pm i \pi$.

The geodesics $\tilde{\partial}_{i}$ and $\tilde{\partial}_{i j}$ for $i, j=1,2,3$ and $i \neq j$ form a right-angled hexagon. The widths of this hexagon are by definition the numbers $l_{i} / 2$ and $l_{i j}$. It is well known Fenchel, W. (1989) that up to a rigid motion the hexagon is determined by the widths $l_{1}, l_{2}, l_{3}$. Therefore the class $[\phi] \in X\left(\pi_{1}(P), P S L_{2}(\mathbb{C})\right)$ is determined by $l_{1}, l_{2}, l_{3}$. So it makes sense to define $l_{i j}\left(l_{1}, l_{2}, l_{3}\right)=l_{i j}(\phi)$. When we use this notation, no orientations are specified, so $l_{i j}$ is well defined only up to $\pm i \pi$.

For later purposes we make the following definitions. If $\phi: \pi_{1}(P) \rightarrow \Gamma=\pi_{1}(M)$ is a homomorphism then define $\tilde{v}_{i j} \in T_{1}\left(\mathbb{H}^{3}\right)$ to be the unit vector based at the point of intersection between $\tilde{\partial}_{i}$ and $\tilde{\partial}_{i j}$ and pointing in the same direction as $\tilde{\partial}_{i j}$ (i.e. towards $\tilde{\partial}_{j}$ ). Let $v_{i j}(\phi)$ be the projection of $\tilde{v}_{i j}$ to $T_{1} M$.

By definition $X_{\{P\}}\left(\pi_{1}(P), \Gamma\right)$ is the set of those $[\phi]$ in $X\left(\pi_{1}(P), \Gamma\right)$ for which $l_{i}(\phi)$ and $l_{i j}(\phi)$ are defined.

Lemma 4.1 (Asymptotics for Pants). For $i=1,2,3$ let $l_{i}: \mathbb{R} \rightarrow \mathbb{F}$ be continuous functions such that $l_{i}(t) \rightarrow \infty$ as $t \rightarrow \infty$. Let $l_{12}=l_{12}\left(l_{1}, l_{2}, l_{3}\right)$. Then

$$
\begin{aligned}
\cosh \left(l_{12}\right)= & 1+2 e^{-l_{1}}+2 e^{-l_{2}}+2 e^{\left(-l_{1}-l_{2}+l_{3}\right) / 2} \\
& +O\left(e^{-2 l_{1}}+e^{-2 l_{2}}+e^{\left(-l_{1}-3 l_{2}+l_{3}\right) / 2}+e^{\left(-3 l_{1}-l_{2}+l_{3}\right) / 2}\right) .
\end{aligned}
$$

Proof. This follows from an application of the law of cosines Fenchel, W. (1989) to the right-angled hexagon formed from the geodesics $\tilde{\partial}_{i}$ and $\tilde{\partial}_{i j}$. Specifically, we obtain

$$
\cosh \left(l_{12}\right)=\frac{\cosh \left(l_{3} / 2\right)+\cosh \left(l_{1} / 2\right) \cosh \left(l_{2} / 2\right)}{\sinh \left(l_{1} / 2\right) \sinh \left(l_{2} / 2\right)}
$$

from which the asymptotics follow.

\section{Pulling on Pants Two Legs at a Time}

For hyperbolic elements $g_{1}, g_{2} \in G$ let $X_{\{P\}}\left(\pi_{1}(P), G ; g_{1}, g_{2}\right)$ be the set of nondegenerate characters $[\phi]$ such that $\phi\left(\left[\partial_{i}^{\prime}\right]\right)$ is conjugate to $g_{i}$ for $i=1,2$. Similarly, if $g_{1}, g_{2} \in \Gamma$ let $X_{\{P\}}\left(\pi_{1}(P), \Gamma ; g_{1}, g_{2}\right)$ 
be the set of nondegenerate characters $[\phi] \in X_{\{P\}}\left(\pi_{1}(P), \Gamma\right)$ such that $\phi\left(\left[\partial_{i}^{\prime}\right]\right)$ is $\Gamma$-conjugate to $g_{i}$ for $i=1,2$. There is a projection map $\pi: X_{\{P\}}\left(\pi_{1}(P), \Gamma ; g_{1}, g_{2}\right) \rightarrow X_{\{P\}}\left(\pi_{1}(P), G ; g_{1}, g_{2}\right)$.

We put coordinates on $X_{\{P\}}\left(\pi_{1}(P), G ; g_{1}, g_{2}\right)$ as follows. Fix $i=1,2$. If $[\phi] \in X_{\{P\}}\left(\pi_{1}(P), G ; g_{1}, g_{2}\right)$ then by conjugating we may assume that $\phi\left(\left[\partial_{i}^{\prime}\right]\right)=g_{i}$. Let $\tilde{v}_{i j}$ be as in the previous section. Recall that $\tilde{v}_{i j} \in T_{1}^{\perp}\left(\operatorname{axis}\left(g_{i}\right)\right)$ since axis $\left(g_{i}\right)=\tilde{\partial}_{i}$. But $\tilde{v}_{i j}$ is determined only up to left multiplication by $g_{i}$. But we will ignore this in order to simplify the notation. The map

$$
[\phi] \rightarrow\left(\tilde{v}_{12}, \tilde{v}_{21}, l_{3}(\phi)\right) \in T_{1}^{\perp}\left(\operatorname{axis}\left(g_{1}\right)\right) \times T_{1}^{\perp}\left(\operatorname{axis}\left(g_{2}\right)\right) \times \mathbb{F}
$$

gives coordinates on $X_{\{P\}}\left(\pi_{1}(P), G ; g_{1}, g_{2}\right)$.

Let $\lambda=\lambda_{g_{1}, g_{2}}$ be the measure on $X_{\{P\}}\left(\pi_{1}(P), G ; g_{1}, g_{2}\right)$ with derivative

$$
d \lambda=\frac{2^{n-3} e^{(n-1) \Re\left(L-l_{1}-l_{2}\right) / 2}}{(2 \pi)^{n-2} \operatorname{vol}\left(T_{1} M\right)} d \operatorname{vol}_{T_{1}^{\perp}\left(\operatorname{axis}\left(g_{1}\right)\right)} d v \operatorname{vol}_{T_{1}^{\perp}\left(\operatorname{axis}\left(g_{2}\right)\right)} d L
$$

where $l_{i}=\operatorname{len}\left(g_{i}\right)$.

Theorem 5.1. Let $g_{1}=g_{1}(t), g_{2}=g_{2}(t) \in \Gamma$. If $n=2$ let $\epsilon>0$. If $n=3$, let $\epsilon \in \mathbb{A}$ be such that $\Re(\epsilon)>0$ and $\Im(\epsilon)>0$. For $i=1,2$ let $l_{i}=\operatorname{len}\left(g_{i}\right)$ and $L=L(t) \in \mathbb{F}$. If $L-l_{1}-l_{2} \rightarrow \infty$ as $t \rightarrow \infty$,

$$
E_{L}=\mathcal{F}_{1} \times \mathcal{F}_{2} \times B(L, \epsilon) \subset T_{1}^{\perp}\left(\operatorname{axis}\left(g_{1}\right)\right) \times T_{1}^{\perp}\left(\operatorname{axis}\left(g_{2}\right)\right) \times \mathbb{F}
$$

and $\mathcal{F}_{i}=\mathcal{F}_{i}^{\prime} \times \sigma_{i}, \mathcal{F}_{i}^{\prime}$ is an arc of $S^{n-2}$ with length $\left(\mathcal{F}_{i}^{\prime}\right) e^{\Re\left(L-l_{1}-l_{2}\right) / 4} \rightarrow \infty$ and $\sigma_{i}$ is a segment of axis $\left(g_{i}\right)$ with length $\left(\sigma_{i}\right) e^{\Re\left(L-l_{1}-l_{2}\right) / 4} \rightarrow \infty$ then $\left|\pi^{-1}\left(E_{t}\right)\right| \sim \lambda_{g_{1}(t), g_{2}(t)}\left(E_{t}\right)$ as $t \rightarrow \infty$.

Proof. By lemma 4.1 if $l_{1}, l_{2}, l_{3}, l_{3}-l_{2}-l_{1}$ all tend to infinity then

$$
l_{12}\left(l_{1}, l_{2}, l_{3}\right)-\left(\left(l_{3}-l_{1}-l_{2}\right) / 2+\ln (4)\right) \rightarrow 0 .
$$

By the previous section and theorem 3.1 if $n=3$ then

$$
\begin{aligned}
\left|\pi^{-1}\left(\mathcal{F}_{1} \times \mathcal{F}_{2} \times B(L, \epsilon)\right)\right| & =\chi\left(\mathcal{F}_{1} \times \mathcal{F}_{2} \times l_{12}\left(l_{1}, l_{2}, B(L, \epsilon)\right)\right. \\
& \sim \chi^{\prime}\left(\mathcal{F}_{1} \times \mathcal{F}_{2} \times B\left(\left(L-l_{1}-l_{2}\right) / 2+\ln (4), \epsilon / 2\right)\right) \\
& \sim \frac{2|\Im(\epsilon)|}{(2 \pi)^{n-2}} \frac{2^{n-1} \sinh ((n-1) \Re(\epsilon) / 2) e^{(n-1) \Re\left(L-l_{1}-l_{2}\right) / 2} \operatorname{vol}\left(\mathcal{F}_{1}\right) \operatorname{vol}\left(\mathcal{F}_{2}\right)}{(n-1) \operatorname{vol}\left(T_{1} M\right)} .
\end{aligned}
$$

A routine calculation shows that this equals $\lambda(E)$. The $n=2$ case is similar.

\section{Pulling on Pants One Leg at a Time}

If $h \in G$ is hyperbolic let $X_{\{P\}}\left(\pi_{1}(P), G ; h\right)$ be the set of nondegenerate characters $[\phi]$ such that $\phi\left(\left[\partial_{1}^{\prime}\right]\right)$ is conjugate to $h$. Similarly, if $h \in \Gamma$ let $X_{\{P\}}\left(\pi_{1}(P), \Gamma ; h\right)$ be the set of nondegenerate characters $[\phi] \in X_{\{P\}}\left(\pi_{1}(P), \Gamma\right)$ such that $\phi\left(\left[\partial_{1}^{\prime}\right]\right)$ is $\Gamma$-conjugate to $h$. There is a projection map $\pi: X_{\{P\}}\left(\pi_{1}(P), \Gamma ; h\right) \rightarrow X_{\{P\}}\left(\pi_{1}(P), G ; h\right)$. 
We put coordinates on $X_{\{P\}}\left(\pi_{1}(P), G ; h\right)$ as follows. Let $\tilde{\eta}=$ axis $(h)$ oriented from the repelling to the attracting fixed point of $h$. Let $\tilde{\sigma}_{2}$ be an oriented geodesic perpendicular to axis $(h)$. The reason for the subscript will be apparent later. If $[\phi] \in X_{\{P\}}\left(\pi_{1}(P), G ; h\right)$ then by conjugating we may assume that $\phi\left(\left[\partial_{1}^{\prime}\right]\right)=h$. Let $\tilde{v}_{12}$ be as in section 4 . Recall that $\tilde{v}_{12} \in T_{1}^{\perp}(\tilde{\eta})$. But $\tilde{v}_{12}$ is determined only up to left multiplication by $h$. Define $y: X_{\{P\}}\left(\pi_{1}(P), G ; h\right) \rightarrow \mathbb{F}$ by $y=$ width $\left(\tilde{\sigma}_{2}, \tilde{v}_{12} ; \tilde{\eta}\right)$ where we have abused notation by identifying $\tilde{v}_{12}$ with the oriented geodesic tangent to it.

The map $C: X_{\{P\}}\left(\pi_{1}(P), G ; h\right) \rightarrow \mathbb{F}^{2} \times \mathbb{F} / \operatorname{len}(h)$ defined by $C(\phi)=\left(l_{2}(\phi), l_{3}(\phi), y(\phi)\right)$ determines the desired coordinates. To avoid excessive notation, we identify $X_{\{P\}}\left(\pi_{1}(P), G ; h\right)$ with $\mathbb{F}^{2} \times \mathbb{F} /$ len $(h)$ through the map $C$. Let $\lambda=\lambda_{h}$ be the measure on $X_{\{P\}}\left(\pi_{1}(P), G ; h\right)$ with derivative

$$
d \lambda=\frac{1}{(2 \pi)^{n-2}} \frac{\exp \left(\left(\frac{n-1}{2}\right) \Re\left(l_{2}+l_{3}-\operatorname{len}(h)\right)\right)}{\operatorname{vol}\left(T_{1}(M)\right)} d l_{2} d l_{3} d y .
$$

Theorem 6.1. If $n=2$ let $\epsilon>0$. If $n=3$, let $\epsilon \in \mathbb{A}$ such that $\Re(\epsilon)>0$ and $\pi \geq \Im(\epsilon)>0$. Let $h=h(t) \in \Gamma, l_{1}=$ len $(h)$. Let $l_{2}, l_{3}, Y: \mathbb{R} \rightarrow \mathbb{F}$ be functions of $t$. Let $x=l_{12}\left(l_{1}, l_{2}, l_{3}\right)$. Suppose that

$$
E_{t}=B\left(l_{2}, \epsilon\right) \times B\left(l_{3}, \epsilon\right) \times B(0, Y) \subset \mathbb{F}^{3},
$$

$l_{2}+l_{3}-l_{1} \rightarrow \infty, \Re\left(l_{3}\right) \geq \Re\left(l_{2}\right)$,

$$
\begin{aligned}
& |\Re(\sinh (x) \sinh (Y))| e^{\Re\left(l_{2}\right) / 2} \rightarrow \infty \text { and in case } n=3 \text { then } \\
& |\Im(\sinh (x) \sinh (Y))| e^{\Re\left(l_{2}\right) / 2} \rightarrow \infty .
\end{aligned}
$$

Then $\left|\pi^{-1}\left(E_{t}\right)\right| \sim \lambda_{h(t)}\left(E_{t}\right)$.

To prove the theorem, we need to introduce more notation. All quantities and geodesics below depend implicitly on a parameter $t$. We will derive formulae which are asymptotic in $t$. Let $[\phi] \in X_{\{P\}}\left(\pi_{1}(P), \pi_{1}(M) ; h\right)$.

Let $g=\phi\left[\partial_{2}^{\prime}\right]$. Let $\tilde{\sigma}_{1}=g^{-1} \tilde{\sigma}_{2}$ and $\tilde{\sigma}_{3}=g \tilde{\sigma}_{2}$. See figure 2 2 For $i=1,2$ let $\tilde{\gamma}_{i}$ be the segment perpendicular to $\tilde{\sigma}_{i}$ and $\tilde{\sigma}_{i+1}$. Let $\tilde{\partial}_{12}$ be perpendicular to $\tilde{\eta}$ and axis $(g)$. Let $\tau$ be perpendicular to $\tilde{\sigma}_{2}$ and $\operatorname{axis}(g)$. Define

$$
\begin{array}{lll}
x=l_{12}(\phi)=w i d t h\left(\eta, \operatorname{axis}(g) ; \tilde{\partial}_{12}\right) & y=\operatorname{width}\left(\tilde{\sigma}_{2}, \tilde{\partial}_{12} ; \tilde{\eta}\right) & z=\operatorname{width}\left(\tilde{\sigma}_{2}, \operatorname{axis}(g) ; \tau\right) \\
u_{1}=w_{i d t h}\left(\tilde{\eta}, \tilde{\gamma}_{1} ; \tilde{\sigma}_{2}\right) & u_{2}=\operatorname{width}\left(\tilde{\eta}, \tilde{\gamma}_{2} ; \tilde{\sigma}_{2}\right) & l_{2}^{\prime}=\operatorname{len}(\gamma)=\operatorname{width}\left(\tilde{\sigma}_{1}, \tilde{\sigma}_{2} ; \tilde{\gamma}_{1}\right) \\
m=\frac{u_{1}+u_{2}}{2} & w=u_{2}-u_{1} & l_{i}=l_{i}(\phi) .
\end{array}
$$

Lemma 6.2. If $l_{1}, l_{2}, l_{3} \rightarrow \infty, l_{2}+l_{3}-l_{1} \rightarrow+\infty, \Re\left(l_{3}\right) \geq \Re\left(l_{2}\right)$ and $\tanh (x) \sinh (y) \rightarrow 0$ as $t \rightarrow \infty$ then

$$
\begin{aligned}
l_{2}^{\prime}-l_{2} & \rightarrow 0 \\
m & \sim x \sim \operatorname{arccosh}\left(1+2 e^{\left(l_{3}-l_{1}-l_{2}\right) / 2}\right) \\
w & \sim-2 \sinh (m) \sinh (y) .
\end{aligned}
$$




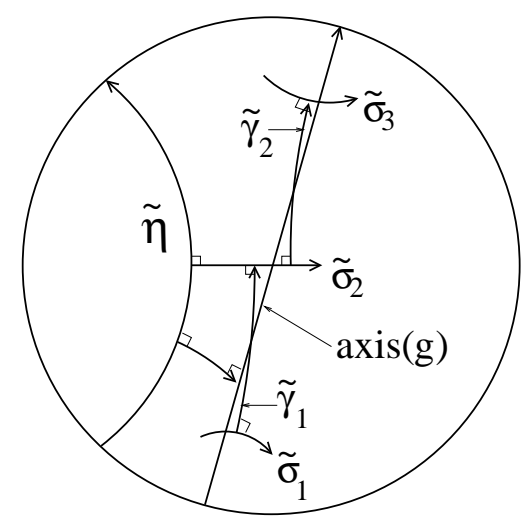

Figure 2:

After this lemma is proven, the theorem will follow from theorem 3.1 and a change of variables. We need some intermediate lemmas.

Lemma 6.3. If $w \rightarrow 0$ and $l_{2} \rightarrow \infty$ (as $\left.t \rightarrow \infty\right)$ then $\operatorname{len}(g)-\operatorname{len}(\gamma) \rightarrow 0$ (i.e. $l_{2}-l_{2}^{\prime} \rightarrow 0$ ) and $z-i \pi / 2 \sim-i w / 2$.

Proof. Recall that a right-angled hexagon is an ordered 6 -tuplet of oriented geodesics $\left(\tilde{H}_{1}, . ., \tilde{H}_{6}\right)$ such that for all $i \bmod 6, \tilde{H}_{i}$ is perpendicular to $\tilde{H}_{i+1}$ Fenchel, W. (1989)]. Let $H=\left(\tilde{H}_{1}, \ldots, \tilde{H}_{6}\right)$ be the right-angled hexagon satisfying

$$
\tilde{H}_{1}=\tilde{\gamma}_{1}, \quad \tilde{H}_{2}=\tilde{\sigma}_{2}, \quad \tilde{H}_{3}=\tau, \quad \tilde{H}_{4}=\operatorname{axis}(g), \quad \tilde{H}_{5}=-\left(g^{-1} \tau\right), \quad \tilde{H}_{6}=\tilde{\sigma}_{1} .
$$

See figure 3, Let $H_{i}:=w i d t h\left(\tilde{H}_{i}, \tilde{H}_{i+2} ; \tilde{H}_{i+1}\right)$ (subscripts mod 6). We claim that

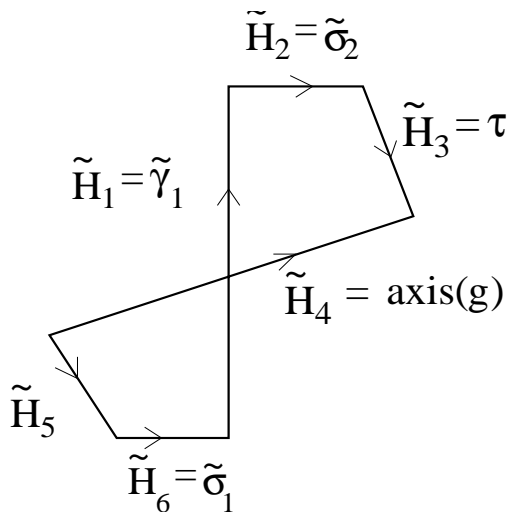

Figure 3: A nongeometric diagram of hexagon $H$.

$$
\begin{array}{lll}
H_{1}=\operatorname{len}(\gamma) & H_{2}=w / 2+i \pi / 2 & H_{3}=z \\
H_{4}=-\operatorname{len}(g)+i \pi & H_{5}=z & H_{6}=w / 2+i \pi / 2 .
\end{array}
$$


The values of $H_{1}$ and $H_{3}$ follow by definition. $g$ maps $\tilde{H}_{4}$ to itself, $\tilde{H}_{5}$ to $-\tilde{H}_{3}$ and $\tilde{H}_{6}$ to $\tilde{H}_{2}$. Since

$$
\text { width }\left(g H_{i}, g H_{j} ; g H_{k}\right)=\operatorname{width}\left(H_{i}, H_{j} ; H_{k}\right)
$$

for any $i, j, k$ it follows that

$$
H_{5}=\operatorname{width}\left(\tilde{H}_{4}, \tilde{H}_{6} ; \tilde{H}_{5}\right)=\operatorname{width}\left(\tilde{H}_{4}, \tilde{H}_{2} ;-\tilde{H}_{3}\right)=\operatorname{width}\left(\tilde{H}_{2}, \tilde{H}_{4} ; \tilde{H}_{3}\right)=H_{3}=z .
$$

Since $g$ maps $\tilde{H}_{5}$ to $-\tilde{H}_{3}$ and $\operatorname{axis}(g)=\tilde{H}_{4}$,

$$
\operatorname{len}(g)=\operatorname{width}\left(\tilde{H}_{5},-\tilde{H}_{3} ; \tilde{H}_{4}\right)=\operatorname{width}\left(\tilde{H}_{5}, \tilde{H}_{3} ; \tilde{H}_{4}\right)+i \pi=-H_{4}+i \pi \text {. }
$$

Hence $H_{4}=-l e n(g)+i \pi$. Observe that

$$
\begin{aligned}
w & =u_{2}-u_{1}=\text { width }\left(\tilde{\gamma}_{1}, \tilde{\gamma}_{2} ; \tilde{\sigma}_{2}\right)=\text { width }\left(\tilde{\gamma}_{1}, \tau ; \tilde{\sigma}_{2}\right)+\text { width }\left(\tau, \tilde{\gamma}_{2} ; \tilde{\sigma}_{2}\right) \\
& =\operatorname{width}\left(\tilde{H}_{1}, \tilde{H}_{3} ; \tilde{H}_{2}\right)+w i d t h\left(g^{-1} \tau, g^{-1} \tilde{\gamma}_{2} ; g^{-1} \tilde{\sigma}_{2}\right) \\
& =H_{2}+\operatorname{width}\left(-\tilde{H}_{5}, \tilde{H}_{1} ; \tilde{H}_{6}\right)=H_{2}+H_{6}+i \pi
\end{aligned}
$$

The law of sines implies

$$
\frac{\sinh \left(H_{3}\right)}{\sinh \left(H_{6}\right)}=\frac{\sinh \left(H_{5}\right)}{\sinh \left(H_{2}\right)}
$$

Since $H_{3}=H_{5}$ this implies $\sinh \left(H_{6}\right)=\sinh \left(H_{2}\right)$. Now we can use the law of cosines to obtain $\cosh \left(H_{6}\right)=\cosh \left(H_{2}\right)$. Together these imply $H_{2}=H_{6}$. Thus, $H_{2}=H_{6}=w / 2 \pm i \pi / 2$. From the figure, it can be checked that $H_{2}=H_{6}=w / 2+i \pi / 2$. This proves the claim.

By the law of cosines,

$$
\cosh \left(H_{4}\right)=\cosh \left(H_{2}\right) \cosh \left(H_{6}\right)+\sinh \left(H_{2}\right) \sinh \left(H_{6}\right) \cosh \left(H_{1}\right) .
$$

Substitution yields

$$
\cosh (-\operatorname{len}(g)+i \pi)=\cosh ^{2}(w / 2+i \pi / 2)+\sinh ^{2}(w / 2+i \pi / 2) \cosh (l e n(\gamma)) .
$$

This simplifies to

$$
\begin{aligned}
\cosh (\operatorname{len}(g)) & =\cosh (\operatorname{len}(\gamma))+\sinh ^{2}(w / 2)(1+\cosh (\operatorname{len}(\gamma))) \\
& =\cosh (\operatorname{len}(\gamma))+O\left(w^{2} e^{\operatorname{len}(\gamma)}\right) .
\end{aligned}
$$

This implies $\operatorname{len}(g)=\operatorname{len}(\gamma)+O\left(w^{2}\right)$. By the law of cosines

$$
\cosh \left(H_{3}\right)=\cosh \left(H_{1}\right) \cosh \left(H_{5}\right)+\sinh \left(H_{1}\right) \sinh \left(H_{5}\right) \cosh \left(H_{6}\right) .
$$

Substitution yields

$$
\cosh (z)=\cosh (\text { len }(\gamma)) \cosh (z)+\sinh (\operatorname{len}(\gamma)) \sinh (z) \cosh (w / 2+i \pi / 2)
$$

By solving for $\operatorname{coth}(z)$ we obtain

$$
\operatorname{coth}(z)=\frac{\sinh (\operatorname{len}(\gamma)) \cosh (w / 2+i \pi / 2)}{1-\cosh (\operatorname{len}(\gamma))}
$$

Since $l_{2}=\operatorname{len}(g) \rightarrow \infty, \operatorname{len}(\gamma) \rightarrow \infty$. So $z-i \pi / 2 \sim-i w / 2$. 
Lemma 6.4. If $\tanh (x) \sinh (y) \rightarrow 0$ and $l_{2} \rightarrow \infty$ then $w \rightarrow 0, x \sim m$ and $w \sim-2 \sinh (m) \sinh (y)$. Proof. Let $P=\left(\tilde{P}_{1}, . ., \tilde{P}_{5}\right)$ be the right-angled pentagon defined by

$$
\tilde{P}_{1}=\tilde{\sigma}_{2}, \quad \tilde{P}_{2}=\tau, \quad \tilde{P}_{3}=\operatorname{axis}(g), \quad \tilde{P}_{4}=\tilde{\partial}_{12}, \quad \tilde{P}_{5}=\tilde{\eta} .
$$

See figure 4. Let $P_{i}:=\left(\tilde{P}_{i-1}, \tilde{P}_{i+1} ; \tilde{P}_{i}\right)$ (subscripts mod 5). We claim

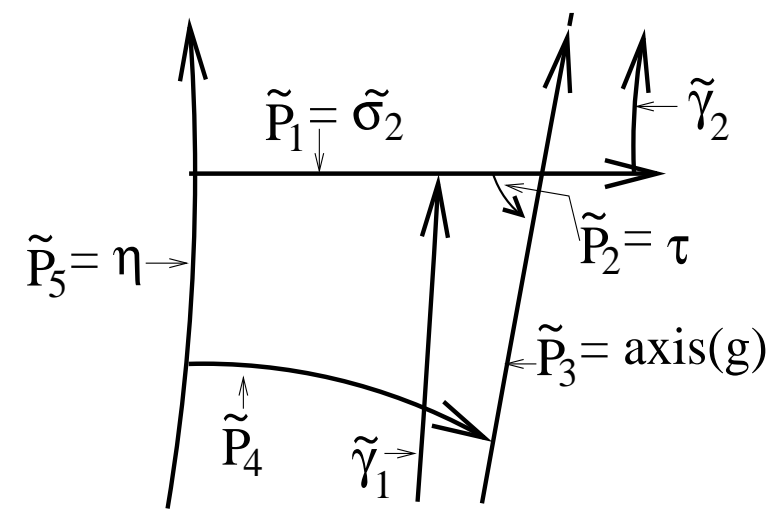

Figure 4: A diagram of pentagon $P$.

$$
P_{1}=m+i \pi / 2, \quad P_{2}=z, \quad P_{4}=-x, \quad P_{5}=-y .
$$

The last three identities hold by definition. The first follows from

$$
\begin{aligned}
P_{1} & =\operatorname{width}\left(\tilde{\eta}, \tau ; \tilde{\sigma}_{2}\right)=w i d t h\left(\tilde{\eta}, \tilde{\gamma}_{1} ; \tilde{\sigma}_{2}\right)+w i d t h\left(\tilde{\gamma}_{1}, \tau ; \tilde{\sigma}_{2}\right) \\
& =u_{1}+H_{2}=u_{1}+w / 2+i \pi / 2=u_{1}+\frac{u_{2}-u_{1}}{2}+i \pi / 2=m+i \pi / 2
\end{aligned}
$$

By the trigonometric formulae for right angled pentagons Fenchel, W. (1989)

$$
\cosh \left(P_{4}\right)=-\sinh \left(P_{1}\right) \sinh \left(P_{2}\right) .
$$

Substituting yields $\cosh (-x)=-\sinh (m+i \pi / 2) \sinh (z)$. Equivalently,

$$
\sinh (z)=\frac{i \cosh (x)}{\cosh (m)}
$$

By other trigonometric formulae for right-angled pentagons Fenchel, W. (1989),

$$
\begin{aligned}
\cosh (z) & =\cosh \left(P_{2}\right)=-\sinh \left(P_{5}\right) \sinh \left(P_{4}\right) \\
& =-\sinh (-y) \sinh (-x)=-\sinh (x) \sinh (y) .
\end{aligned}
$$

Dividing equation 9 by equation 8 yields $\operatorname{coth}(z)=i \tanh (x) \sinh (y) \cosh (m)$. With equation 7 this implies

$$
i \tanh (x) \sinh (y)=-\frac{\sinh (\operatorname{len}(\gamma)) \operatorname{sech}(m) \cosh (w / 2+i \pi / 2)}{\cosh (\operatorname{len}(\gamma))-1)}
$$


By hypothesis $\tanh (x) \sinh (y) \rightarrow 0$. Since $\frac{\sinh (\operatorname{len}(\gamma))}{\cosh (\operatorname{len}(\gamma))-1}$ and $\operatorname{sech}(m)$ are bounded from below, this implies that $\sinh (w) \rightarrow 0$. So $w \rightarrow 0$. This allows us to use results from the previous lemma.

Since $\sinh (z) \rightarrow i$ and $\sinh (m+i \pi / 2)=i \cosh (m)$ equation 8 implies $\cosh (x)-1 \sim \cosh (m)-1$. This implies $x \sim \pm m$. From the figure it can be seen that $x \sim m$. By equation 9

$$
\sinh (y)=\frac{-\cosh (z)}{\sinh (x)} \sim \frac{-\cosh (-i w / 2+i \pi / 2)}{\sinh (m)}=\frac{i \sinh (i w / 2)}{\sinh (m)} \sim \frac{-w}{2 \sinh (m)} .
$$

So $w \sim-2 \sinh (m) \sinh (y)$.

Proof. (of lemma 6.2) By lemma 6.4 $w \rightarrow 0$ so we can use lemma 6.3. So $l_{2}^{\prime}-l_{2} \rightarrow 0$. Since $l_{2}+l_{3}-l_{1} \rightarrow \infty, \Re\left(l_{3}\right) \geq \Re\left(l_{2}\right)$ and $l_{1}, l_{2}, l_{3} \rightarrow \infty$ it follows from lemma 4.1 that

$$
\cosh \left(l_{12}(\phi)\right)-1 \sim 2 e^{\left(l_{3}-l_{1}-l_{2}\right) / 2} .
$$

By definition, $x=l_{12}(\phi)$. By lemma $6.4 x \sim m$ so $\cosh (m)-1 \sim 2 e^{\left(l_{3}-l_{1}-l_{2}\right) / 2}$ too. By lemma 6.4 $w \sim-2 \sinh (m) \sinh (y)$.

Proof. (of theorem 6.1)

Let $\eta$ be the projection of $\tilde{\eta}$ to $M=\mathbb{H}^{n} / \Gamma$. Let $\sigma$ be the projection of $\tilde{\sigma}_{2}$ to $M$.

Let $\chi=\chi_{\sigma, \sigma}$ be as defined in theorem 3.1. Recall that $\chi$ is a measure on $T_{1}^{\perp}(\sigma) \times T_{1}^{\perp}(\sigma) \times \mathbb{F}$. Indeed $\chi(E)$ is the number of oriented perpendiculars $\gamma$ from $\sigma$ to $\sigma$ such that $\left(v_{1}, v_{2}, \operatorname{len}(\gamma)\right) \in E$ where $v_{i}$ is the $i$-th endpoint vector of $\gamma$. It is convenient for the proof to put coordinates on $T_{1}^{\perp}(\sigma)$. So define $u: T_{1}^{\perp}(\sigma) \rightarrow \mathbb{F}$ by $u(v)=w i d t h(\eta, v ; \sigma)$. We have abused notation here by identifying $v$ with the oriented geodesic tangent to it. Define $K: T_{1}^{\perp}(\sigma)^{2} \times \mathbb{F} \rightarrow \mathbb{F}^{3}$ by $K\left(v_{1}, v_{2}, l_{2}\right)=$ $\left(l_{2}, u\left(v_{1}\right), u\left(v_{2}\right)\right)$.

Let $\rho$ be the density of $K_{*} \chi^{\prime}$. From the definition of $\chi^{\prime}$ (see the paragraphs before theorem 3.1) it follows that

$$
\rho\left(L, u_{1}, u_{2}\right)=\frac{e^{(n-1) \Re(L)}}{(2 \pi)^{n-2} 2^{n-1} \operatorname{vol}\left(T_{1} M\right)} .
$$

The parameters $l_{1}, l_{2}, l_{3}, y$ determine $\tilde{\eta}, \tilde{\sigma}_{2}, \operatorname{axis}(g), \tilde{\gamma}_{i}$, etc. up to a rigid motion. Hence, keeping $l_{1}$ fixed we may define $G\left(l_{2}, l_{3}, y\right)=\left(l_{2}^{\prime}, m, w\right)$. Define

$$
H\left(l_{2}^{\prime}, m, w\right)=\left(l_{2}^{\prime}, u_{1}, u_{2}\right)=\left(l_{2}^{\prime}, m-\frac{w}{2}, m+\frac{w}{2}\right) .
$$

By definition $\lambda\left(E_{t}\right)=\left(K_{*} \chi\right)\left(H \circ G\left(E_{t}\right)\right)$. It follows from theorem 3.1 that $\left(K_{*} \chi\right)\left(H \circ G\left(E_{t}\right)\right) \sim$ $\left(K_{*} \chi^{\prime}\right)\left(H \circ G\left(E_{t}\right)\right)$ once we show that its hypotheses are satisfied. It is immediate that $K^{-1}(H \circ$ $\left.G\left(E_{t}\right)\right)$ tends to infinity with $t$. We need to show that

$$
\lim _{t \rightarrow \infty} \frac{\chi^{\prime}\left(N_{1}\left(1, \partial K^{-1} \circ H \circ G\left(E_{t}\right)\right)\right)}{\left.\chi^{\prime}\left(K^{-1} \circ H \circ G\left(E_{t}\right)\right)\right)}=0 .
$$

By definition

$$
\begin{aligned}
N_{1}\left(1, \partial K^{-1} \circ H \circ G\left(E_{t}\right)\right):= & K^{-1}\left(\left\{\left(u_{1}, u_{2}, l_{2}\right) \in \mathbb{F}^{3} ; \exists\left(u_{1}^{\prime}, u_{2}^{\prime}, l_{2}\right) \in \partial(H \circ G)\left(E_{t}\right)\right.\right. \\
& \text { such that } \left.\left.d\left(u_{1}^{\prime}, u_{1}\right) \leq e^{-\Re\left(l_{2}\right) / 2}, d\left(u_{2}^{\prime}, u_{2}\right) \leq e^{-\Re\left(l_{2} / 2\right)}\right\}\right) .
\end{aligned}
$$


But the Jacobian of the map $\left(u_{1}, u_{2}\right) \rightarrow(m, w)=\left(\frac{u_{1}+u_{2}}{2}, u_{2}-u_{1}\right)$ is constant. So it suffices to show that

$$
\lim _{t \rightarrow \infty} \frac{\chi^{\prime}\left(K^{-1} \circ H\left(N_{1}\left(1, \partial G\left(E_{t}\right)\right)\right)\right)}{\chi^{\prime}\left(K^{-1} \circ H \circ G\left(E_{t}\right)\right)}=0 .
$$

where

$$
\begin{aligned}
N_{1}\left(1, \partial G\left(E_{t}\right)\right):=\quad & \left\{\left(l_{2}, m, w\right) \in \mathbb{F}^{3} ; \exists\left(l_{2}, m^{\prime}, w^{\prime}\right) \in \partial G\left(E_{t}\right)\right. \\
& \text { such that } \left.d\left(m, m^{\prime}\right) \leq e^{-\Re\left(l_{2}\right) / 2}, d\left(w, w^{\prime}\right) \leq e^{-\Re\left(l_{2} / 2\right)}\right\} .
\end{aligned}
$$

By lemma 6.2 $m \sim \operatorname{arccosh}\left(1+2 \exp \left(\left(l_{3}-l_{1}-l_{2}\right) / 2\right)\right)$. Since $l_{3}+l_{2}-l_{1} \rightarrow \infty$ it follows that the range of $\Re(m) e^{\Re\left(l_{2}\right) / 2} \rightarrow \infty$ and, if $n=3$, the range of $\Im(m) e^{\Re\left(l_{2}\right) / 2} \rightarrow \infty$. Since $w \sim-2 \sinh (m) \sinh (y)$, and $\Re(\sinh (m) \sinh (Y)) e^{\Re\left(l_{2}\right) / 2} \rightarrow \infty$ (and if $n=3, \Im(\sinh (m) \sinh (Y)) e^{\Re\left(l_{2}\right) / 2} \rightarrow \infty$ too) by hypothesis, equation 11 is satisfied.

The last hypothesis of theorem 3.1 is easy to verify. So $\left(K_{*} \chi\right)\left(H \circ G\left(E_{L}\right)\right) \sim\left(K_{*} \chi^{\prime}\right)\left(H \circ G\left(E_{L}\right)\right)$. Define

$$
\tilde{G}\left(l_{2}, l_{3}, y\right)=\left(l_{2}, \operatorname{arccosh}\left(1+2 e^{\left(l_{3}-l_{1}-l_{2}\right) / 2}\right),-2 \sinh \left(\operatorname{arccosh}\left(1+2 e^{\left(l_{3}-l_{1}-l_{2}\right) / 2}\right)\right) \sinh (y)\right) .
$$

Lemma 6.2 implies $\tilde{G} \sim G$ provided that $\tanh (x) \sinh (y) \rightarrow 0$ for $y \in B(0, Y)$. This may not be the case, but we can always choose $Y$ smaller if necessary so that it holds. But we cannot choose $Y$ too small since it is necessary to maintain the hypothesis that

$$
\Re(\sinh (x) \sinh (Y)) e^{\Re\left(l_{2}\right) / 2} \rightarrow \infty \text { and if } n=3 \text { then } \Im(\sinh (x) \sinh (Y)) e^{\Re\left(l_{2}\right) / 2} \rightarrow \infty, \text { too. }
$$

It is easy to see that this can be achieved.

Now that we have $G \sim \tilde{G}$ it can easily be verified that $\left(K_{*} \chi^{\prime}\right)\left(H \circ G\left(E_{L}\right)\right) \sim\left(K_{*} \chi^{\prime}\right)\left(H \circ \tilde{G}\left(E_{L}\right)\right)$. So we have shown that $\left|\pi^{-1}\left(E_{t}\right)\right| \sim\left(K_{*} \chi^{\prime}\right)\left(H \circ \tilde{G}\left(E_{L}\right)\right)$. To finish we need only compute the later:

$$
\begin{aligned}
\left(K_{*} \chi^{\prime}\right)\left(H \circ \tilde{G}\left(E_{t}\right)\right) & =\int_{H \circ G\left(E_{t}\right)} \rho\left(l_{2}, u_{1}, u_{2}\right) d l_{2} d u_{1} d u_{2} \\
& \sim \int_{E_{t}} \rho\left(H \circ \tilde{G}\left(l_{2}, l_{3}, y\right)\right)|\operatorname{Jac}(H \circ \tilde{G})| d l_{2} d l_{3} d y .
\end{aligned}
$$

An easy computation shows that the Jacobian of $H$ is 1 and

$$
|J a c(\tilde{G})|=2^{n-1} e^{(n-1) \Re\left(l_{3}-l_{1}-l_{2}\right) / 2} .
$$

Another short computation shows

$$
\rho\left(H \circ \tilde{G}\left(l_{2}, l_{3}, y\right)\right)=\frac{e^{(n-1) \Re\left(l_{2}\right)}}{(2 \pi)^{n-2} 2^{n-1} \operatorname{vol}\left(T_{1} M\right)} .
$$

The theorem follows from the above three equations. 


\section{Telescoping Paths}

Theorem 7.1. If $F$ is a free group of finite rank and for each $t>0,\left[\phi_{t}\right] \in X_{\{P\}}\left(F, I s o m^{+}\left(\mathbb{H}^{2}\right)\right)$ is a sequence of discrete and faithful characters such that the length of the shortest closed curve on $\mathbb{H}^{2} / \phi_{t}(F)$ tends to infinity then there exists a pants decomposition $\mathcal{P}_{t}$ of the convex core of $\mathbb{H}^{2} / \phi_{t}(F)$ such that $\left\{\left(\left[\phi_{t}\right], \mathcal{P}_{t}\right)\right\}_{t>0}$ is telescoping.

It is possible that this result holds in all dimensions.

Proof. Let $S_{t}$ be the convex core of $\mathbb{H}^{2} / \phi_{t}(F)$. By definition, the convex core is the smallest closed convex subset of $\mathbb{H}^{2} / \phi_{t}(F)$ that is homotopy equivalent to it. It is a compact surface with geodesic boundary.

The proof is by induction on the number of pants in a pants decomposition of $S_{t}$. If $S_{t}$ is a single pair of pants, then the result is vacuous.

Let $\alpha_{t}$ be the shortest homotopically nontrivial arc on $S_{t}$ with both endpoints in the boundary. Let $P_{t} \subset S_{t}$ be the unique pair of pants containing $\alpha_{t}$. If $b_{1}, b_{2}$ are the boundary components containing the endpoints of $\alpha$ then $P_{t}$ is homotopy equivalent to a regular neighborhood of $\alpha_{t} \cup b_{1} \cup b_{2}$. It is possible that $b_{1}=b_{2}$.

Let $S_{t}^{\prime}$ be the closure of $S_{t}-P_{t}$. Let $F^{\prime}$ be the free group with $\operatorname{rank}\left(F^{\prime}\right)=\operatorname{rank}(F)-1$. There exists a representation $\phi_{t}^{\prime}: F^{\prime} \rightarrow I \operatorname{Isom}^{+}\left(\mathbb{H}^{2}\right)$ such that $S_{t}^{\prime}$ is isometric to the convex hull of $\mathbb{H}^{2} / \phi_{t}^{\prime}\left(F^{\prime}\right)$. By induction, we may assume that there exists a pants decomposition $\mathcal{P}_{t}^{\prime}$ of $S_{t}^{\prime}$ for which $\left(\phi_{t}^{\prime}, \mathcal{P}_{t}^{\prime}\right)$ is telescoping. So it suffices to show that $\operatorname{len}\left(\partial S_{t}\right)-\operatorname{len}\left(\partial S_{t}^{\prime}\right) \rightarrow \infty$ as $t \rightarrow \infty$.

We claim that the length of $\alpha_{t}$ tends to zero as $t \rightarrow \infty$. Since the length of the shortest closed geodesic on $S_{t}$ tends to infinity it follows that $\operatorname{len}\left(\partial S_{t}\right) \rightarrow \infty$. Since $\alpha_{t}$ is the shortest nontrivial arc with endpoints in the boundary, it follows that the $\operatorname{len}\left(\alpha_{t}\right) / 2$ neighborhood of the boundary does not have self-intersections. Thus its area is at least $\operatorname{len}\left(\partial S_{t}\right) \operatorname{len}\left(\alpha_{t}\right) / 2$. But the total area of the surface is bounded by $2 \pi(\operatorname{rank}(F)-1)$ by Gauss-Bonet. So len $\left(\partial S_{t}\right) \operatorname{len}\left(\alpha_{t}\right) / 2 \leq 2 \pi(\operatorname{rank}(F)-1)$. Since len $\left(\partial S_{t}\right)$ tends to infinity, it must be that $\operatorname{len}\left(\alpha_{t}\right) \rightarrow 0$.

There are two cases depending on whether $P_{t} \cap S_{t}^{\prime}$ has one or two components.

Case 1: Suppose that $P_{t} \cap S_{t}^{\prime}$ is a single component which we call $\partial_{3}$. Then $\alpha_{t}$ must be the shortest arc between the other two boundary components of $P_{t}$. In the notation of lemma 4.1, the other two components are $\partial_{1}, \partial_{2}$ with lengths $l_{1}\left(\phi_{t}\right), l_{2}\left(\phi_{t}\right)$ and $\alpha_{t}=\partial_{12}$ is the shortest arc from $\partial_{1}$ to $\partial_{2}$. Thus $\operatorname{len}\left(\alpha_{t}\right)=l_{12}$. Thus we have

$$
\begin{aligned}
\cosh \left(\operatorname{len}\left(\alpha_{t}\right)\right)= & 1+2 e^{-l_{1}}+2 e^{-l_{2}}+2 e^{\left(-l_{1}-l_{2}+l_{3}\right) / 2} \\
& +O\left(e^{-2 l_{1}}+e^{-2 l_{2}}+e^{\left(-l_{1}-3 l_{2}+l_{3}\right) / 2}+e^{\left(-3 l_{1}-l_{2}+l_{3}\right) / 2}\right)
\end{aligned}
$$

where $l_{3}$ is the length of $\partial_{3}$. Since len $\left(\alpha_{t}\right)$ tends to zero, it must be that $l_{1}+l_{2}-l_{3}$ tends to infinity. But $l_{1}+l_{2}-l_{3}=\operatorname{len}\left(\partial S_{t}\right)-\operatorname{len}\left(\partial S_{t}^{\prime}\right)$. This finishes case 1 .

Case 2: Suppose that $P_{t} \cap S_{t}^{\prime}$ has two components $\partial_{1}$ and $\partial_{2}$. Let $\partial_{3}$ be the other boundary component. Both endpoints of $\alpha_{t}$ lie in $\partial_{3}$. As in section 4 let $\partial_{i j}$ be the shortest segment from $\partial_{i}$ to $\partial_{j}$. $\alpha_{t}$ is the union of the two shortest paths from $\partial_{3}$ to $\partial_{12}$. Let $l_{i j}$ be the length of $\partial_{i j}$.

Cut the pair of pants $P$ along the segments $\partial_{i j}$ for all $i \neq j$. This decomposes $P$ into two isometric right-angled hexagons in the usual way. Next, cut along $\alpha_{t}$. This decomposes each hexagon into 


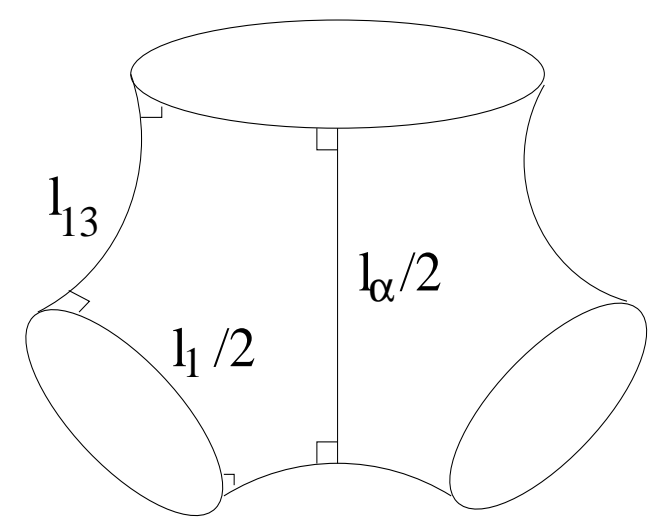

Figure 5: The pairs of pants $P$ with pentagon shown and 3 sides labeled by their lengths.

2 right-angled pentagons one of which has 3 different sides of length $l_{1} / 2, l_{13}$ and $\operatorname{len}\left(\alpha_{t}\right) / 2$. Let $l_{\alpha}:=\operatorname{len}\left(\alpha_{t}\right)$. See figure 5 ,

From the trigonometric formulas for right-angled pentagons [Fenchel, W. (1989)], we have

$$
\cosh \left(l_{\alpha} / 2\right)=\sinh \left(l_{1} / 2\right) \sinh \left(l_{13}\right) .
$$

By the law of cosines

$$
\cosh \left(l_{13}\right)=\frac{\cosh \left(l_{2} / 2\right)}{\sinh \left(l_{1} / 2\right) \sinh \left(l_{3} / 2\right)}+\operatorname{coth}\left(l_{1} / 2\right) \operatorname{coth}\left(l_{3} / 2\right)
$$

Square both sides of equation 12 to obtain

$$
\begin{aligned}
\cosh ^{2}\left(l_{\alpha} / 2\right)= & \sinh ^{2}\left(l_{1} / 2\right)\left(\cosh ^{2}\left(l_{13}\right)-1\right) \\
= & \frac{\sinh ^{2}\left(l_{1} / 2\right) \cosh ^{2}\left(l_{2} / 2\right)}{\sinh ^{2}\left(l_{1} / 2\right) \sinh ^{2}\left(l_{3} / 2\right)} \\
& +2 \frac{\sinh ^{2}\left(l_{1} / 2\right) \cosh \left(l_{2} / 2\right) \operatorname{coth}\left(l_{1} / 2\right) \operatorname{coth}\left(l_{3} / 2\right)}{\sinh \left(l_{1} / 2\right) \sinh \left(l_{3} / 2\right)} \\
& +\left(\operatorname{coth}^{2}\left(l_{1} / 2\right) \operatorname{coth}^{2}\left(l_{3} / 2\right)-1\right) \sinh ^{2}\left(l_{1} / 2\right) .
\end{aligned}
$$

The last term above equals

$$
\cosh ^{2}\left(l_{1} / 2\right) \operatorname{coth}^{2}\left(l_{3} / 2\right)-\sinh ^{2}\left(l_{1} / 2\right)=\sinh ^{2}\left(l_{1} / 2\right)\left(\operatorname{coth}^{2}\left(l_{3} / 2\right)-1\right)+\operatorname{coth}^{2}\left(l_{3} / 2\right) \geq 1 .
$$

Since $\cosh ^{2}\left(l_{\alpha} / 2\right) \rightarrow 1$ the other terms in equation 13 tend to zero. In particular,

$$
2 \frac{\sinh ^{2}\left(l_{1} / 2\right) \cosh \left(l_{2} / 2\right) \operatorname{coth}\left(l_{1} / 2\right) \operatorname{coth}\left(l_{3} / 2\right)}{\sinh \left(l_{1} / 2\right) \sinh \left(l_{3} / 2\right)} \rightarrow 0
$$

This implies $l_{3}-l_{1}-l_{2}$ tends to infinity. But $l_{3}-l_{1}-l_{2}=\operatorname{len}\left(\partial S_{t}\right)-\operatorname{len}\left(\partial S_{t}^{\prime}\right)$. This completes case 2 and finishes the theorem. 


\section{Proofs of the Main Theorems}

Proof. (of theorem 1.2) As in the proof of lemma 3.2 we may assume without loss of generality that $E_{t} \subset X_{\mathcal{P}}\left(\pi_{1}(S), G\right)$ is a rectangle with respect to the Fenchel-Nielsen coordinates induced by $\mathcal{P}$.

The proof is by induction on the rank $r$ of the free group $\pi_{1}(S)$. Recall $\mathcal{P}=\left\{P_{i}\right\}_{i=1}^{r-1}$. If $r=2$ let $S^{\prime}=S_{t}^{\prime}$ be a boundary component of $P_{1}$ such that $\Re\left(l_{\phi_{t}}\left(S^{\prime}\right)\right) \leq \Re\left(l_{\phi_{t}}(\gamma)\right)$ for any other boundary component $\gamma$ of $P_{1}$. Otherwise let $S^{\prime}=\cup_{i=1}^{r-2} P_{i}$.

Define the restriction map

$$
R: X_{\mathcal{P}}\left(\pi_{1}(S), G\right) \rightarrow X_{\mathcal{P}^{\prime}}\left(\pi_{1}\left(S^{\prime}\right), G\right) \quad, \quad R([\phi])=\left[\left.\phi\right|_{\pi_{1}\left(S^{\prime}\right)}\right]
$$

where $\mathcal{P}^{\prime}=\left\{P_{i}\right\}_{i=1}^{r-2}$ if $r>2$ and $\mathcal{P}^{\prime}=\emptyset$ otherwise. If $r=2, X_{\mathcal{P}^{\prime}}\left(\pi_{1}\left(S^{\prime}\right), G\right)$ is just $X\left(\pi_{1}\left(S^{\prime}\right), G\right)$. If $r=2$ let $\nu^{\prime}$ be the measure on $X_{\mathcal{P}^{\prime}}\left(\pi_{1}\left(S^{\prime}\right), G\right)$ with derivative

$$
d \nu^{\prime}=\frac{e^{(n-1) \Re\left(\operatorname{len}\left(S^{\prime}\right)\right)}}{(2 \pi)^{n-2 \Re\left(\operatorname{len}\left(S^{\prime}\right)\right)}} \operatorname{dlen}\left(S^{\prime}\right) .
$$

If $r>2$ then let $\nu^{\prime}$ be the measure on $X_{\mathcal{P}^{\prime}}\left(\pi_{1}\left(S^{\prime}\right), G\right)$ with derivative

$$
d \nu^{\prime}=\operatorname{vol}\left(T_{1} M\right)^{2-r} 2^{(n-3)\left(- \text { genus }\left(S^{\prime}\right)\right)}(2 \pi)^{(1-r)(n-2)} e^{\Re\left[l e n_{\psi}(\partial S)\right](n-1) / 2} d \operatorname{vol}_{\mathcal{P}^{\prime}}(\psi) .
$$

We claim $\left|\pi^{-1}\left(R\left(E_{t}\right)\right)\right| \sim \nu^{\prime}\left(R\left(E_{t}\right)\right)$. This follows from the prime geodesic theorem if $r=2$ and from the induction hypothesis if $r>2$.

To simplify notation, let $P$ denote $P_{r-1}$. Suppose $\left[\phi^{\prime}\right] \in X_{\mathcal{P}^{\prime}}\left(\pi_{1}\left(S^{\prime}\right), G\right)$ and $\left[\phi^{\prime \prime}\right] \in X_{\{P\}}\left(\pi_{1}(P), G\right)$ are such that for every $g \in \pi_{1}(S)$ which represents a curve in $P \cap S^{\prime}, \phi^{\prime}(g)$ is conjugate to $\phi^{\prime \prime}(g)$. Then we may form the connected sum

$$
\phi=\phi^{\prime} \#_{P \cap S^{\prime}} \phi^{\prime \prime} \in X_{\mathcal{P}}\left(\pi_{1}(S), G\right)
$$

in the obvious way. For $\phi^{\prime} \in R\left(E_{t}\right)$, let $E_{t, \phi^{\prime}}$ be the set of all $\phi^{\prime \prime} \in X_{\{P\}}\left(\pi_{1}(P), G\right)$ for which $\phi^{\prime} \#_{P \cap S} \phi^{\prime \prime} \in E_{t}$. So $E_{t, \phi^{\prime}}=R^{-1}\left(\phi^{\prime}\right) \cap E_{t}$.

Let $c$ be the number of components of $P \cap S$. If $c=1$ then $P \cap S$ has a single component $\partial_{1}$ and $E_{t, \phi^{\prime}} \subset X_{\{P\}}\left(\pi_{1}(P), G ; \phi^{\prime}\left(\partial_{1}\right)\right)$. If $c=2$ then $P \cap S$ consists of two components $\partial_{1}, \partial_{2}$ and $E_{t, \phi^{\prime}} \subset X_{\{P\}}\left(\pi_{1}(P), G ; \phi^{\prime}\left(\partial_{1}\right), \phi^{\prime}\left(\partial_{2}\right)\right)$. We have abused notation here by identifying $\partial_{1}$ and $\partial_{2}$ with elements of $\pi_{1}(P)$ that represent them.

If $c=1$, let $\lambda=\lambda_{\phi^{\prime}\left(\partial_{1}\right)}$ be as in theorem 6.1. If $c=2$ let $\lambda=\lambda_{\phi^{\prime}\left(\partial_{1}\right), \phi^{\prime}\left(\partial_{2}\right)}$ be as in theorem 5.1, Theorems 6.1 and 5.1 imply that for $\phi_{t}^{\prime} \in R\left(E_{t}\right)$

$$
\left|\pi^{-1}\left(R^{-1}\left(\phi^{\prime}\right) \cap E_{t}\right)\right| \sim \lambda\left(R^{-1}\left(\phi^{\prime}\right) \cap E_{t}\right)=\lambda\left(E_{t, \phi^{\prime}}\right) .
$$

Using the induction hypothesis, this implies

$$
\left|\pi^{-1}\left(E_{t}\right)\right|=\sum_{\left[\phi^{\prime}\right] \in R\left(E_{t}\right)}\left|\pi^{-1}\left(R^{-1}\left(\phi^{\prime}\right) \cap E_{t}\right)\right| \sim \int_{R\left(E_{t}\right)} \lambda\left(E_{t, \phi^{\prime}}\right) d \nu^{\prime}\left(\phi^{\prime}\right) .
$$

A routine calculation shows that this equals $\nu\left(E_{t}\right)$.

Proof. (of Theorem 1.10 It needs only be noted that the sequence $\left\{N_{\epsilon}\left[\phi_{t}\right]\right\}_{t}$ satisfies the hypothesis of theorem 1.2. This is easy and we leave it to the reader. 


\section{References}

[Bekka, M.; Mayer M. (2000)] Ergodic Theory and Topological Dynamics of Group Actions on Homogeneous Spaces, London Math. Soc. Lecture Notes, 269 (2000), Cambridge University Press.

[Casson, A.; Bleiler S. (1988)] Automorphisms of surfaces after Nielsen and Thurston. London Mathematical Society Student Texts, 9. Cambridge University Press, Cambridge, 1988. iv+105 pp.

[Coornaert, M.; Knieper, G. (2002)] Growth of conjugacy classes in Gromov hyperbolic groups. Geom. Funct. Anal. 12, no. 3, 464-478.

[Fenchel, W. (1989)] Elementary geometry in hyperbolic space. With an editorial by Heinz Bauer. de Gruyter Studies in Mathematics, 11. Walter de Gruyter \& Co., Berlin, 1989. xii+225 pp.

[Gunesch, R. (2005)] Precise asymptotics for periodic orbits of the geodesic flow in nonpositive curvature, preprint.

[Huber, H. (1961)] Zur analytischen Theorie hyperbolischer Raumformen und Bewegungsgruppen. II. (German) Math. Ann. 143.

[Hurt, N.E. (2001)] The prime geodesic theorem and quantum mechanics on finite volume graphs: a review. Rev. Math. Phys. 13, no. 12, 1459-1503.

[Knieper, G. (1997)] On the asymptotic geometry of nonpositively curved manifolds. Geom. Funct. Anal. 7 , no. 4, 755-782.

[Margulis, G.A. (2004)] On some aspects of the theory of Anosov systems. With a survey by Richard Sharp: Periodic orbits of hyperbolic flows. Translated from the Russian by Valentina Vladimirovna Szulikowska. Springer Monographs in Mathematics. Springer-Verlag, Berlin. vi+139 pp.

[Mirzakhani, M (2006)] Weil-Petersson Volumes and Intersection Theory on the Moduli Space of Curves. Jour. Amer. Math. Soc., electronically published March 8, 2006.

[Parry W.; Pollicott, M. (1983)] An analogue of the prime number theorem for closed orbits of Axiom A flows. Ann. of Math. (2) 118, no. 3, 573-591.

[Ratner, M. (1991)] M. Ratner, Raghunathan's topological conjecture and distributions of unipotent flows, Duke J. Math. (63), 235-280, 1991.

[Wolpert, S. (1982)] S. Wolpert, The Fenchel-Nielsen deformation, Annals of Math. (115), 1982, 501-528. 